\title{
What is the best combination treatment with transarterial chemoembolization of unresectable hepatocellular carcinoma? a systematic review and network meta-analysis
}

\author{
Hui Xie ${ }^{1,2}$, Haipeng Yu ${ }^{1}$, Shengtao Tian ${ }^{2}$, Xueling Yang ${ }^{1}$, Ximing Wang ${ }^{2}$, Zhao Yang ${ }^{2}$, \\ Huaming Wang ${ }^{2}$ and Zhi Guo ${ }^{1}$ \\ ${ }^{1}$ Department of Interventional Therapy, Tianjin Medical University Cancer Institute and Hospital, National Clinical Research \\ Center for Cancer, Key Laboratory of Cancer Prevention and Therapy, Tianjin's Clinical Research Center for Cancer, Tianjin \\ 300070, China \\ ${ }^{2}$ Department of Interventional Therapy, 302 Hospital of People's Liberation Army, Beijing 100039, China \\ Correspondence to: Zhi Guo, email: gztjzlyy@163.com \\ Huaming Wang, email: hmwang302@126.com
}

Keywords: transarterial chemoembolization, TACE, unresectable hepatocellular carcinoma, combination treatment, network meta-analysis

Received: July 07, $2017 \quad$ Accepted: July 30, $2017 \quad$ Published: August 10, 2017

Copyright: Xie et al. This is an open-access article distributed under the terms of the Creative Commons Attribution License 3.0 (CC BY 3.0), which permits unrestricted use, distribution, and reproduction in any medium, provided the original author and source are credited.

\section{ABSTRACT}

Objective: To assess the comparative efficacy and safety of combination treatment with transarterial chemoembolization (TACE) for patients with unresectable hepatocellular carcinoma (HCC) through a systematic review and network metaanalysis and to identify what is the best combination treatment with TACE.

Materials and Methods: A network meta-analysis was used to identify evidence from relevant randomized controlled trials. We searched databases for publications up to June 2017. The prespecified primary efficacy outcomes were treatment response and 6-month to 3-year overall survival (OS), while the secondary efficacy outcomes were 1- and 2-year disease-free survival (DFS); safety outcomes were advance effects of combination treatment. We conducted pairwise meta-analyses using a randomeffects model and then performed random-effects network meta-analyses.

Results: A total of 48 trials were eligible (50 analyses), involving 5627 patients and 19 treatment arms. In comparison with other types of combination therapy arms, network meta-analysis disclosed that TACE + three-dimensional conformal radiotherapy, TACE + percutaneous ethanol injection, TACE + percutaneous microwave coagulation therapy, TACE + percutaneous acetic acid injection, and TACE + sorafenib were the more effective methods in treatment response, 6-month to 3-year OS, and 1-2 year DFS; the adverse effects of TACE + sorafenib were serious. The study was registered with PROSPERO, number CRD42017071102.

Conclusions: When considering the efficacy, combination therapy with TACE seemed to offer clear advantages for patients with unresectable HCC. TACE + Three-dimensional conformal radiotherapy, TACE + Percutaneous ethanol injection, TACE + Percutaneous microwave coagulation therapy, and TACE + Percutaneous acetic acid injection are likely the best options to consider in the application of combination treatment.

\section{INTRODUCTION}

Hepatocellular carcinoma (HCC) is one of the most common cancers and has the dismal outcome of cancerrelated death [1]. The incidence of HCC has increased dramatically in the past decade, making $\mathrm{HCC}$ the fifth most common cancer in the world currently. Incidence rates continue to increase rapidly for liver cancer, by about $3 \%$ per year in women and $4 \%$ per year in men, although rates have begun to decline in adults younger than 50 years 
of age [2]. It is recognized that only a small proportion of patients with early-stage HCC may benefit from radical options, such as surgical resection and orthotopic liver transplantation. Although hepatic resection offers a firstline hope for patients suffering from HCC, only a small proportion (10-15\%) of HCC patients are suitable [3]. However, surgical resection is not the first treatment choice for HCC patients with large lesions or poor liver function.

Palliative care and management including transarterial chemoembolization (TACE) is prescribed for most HCC patients to prevent and relieve suffering and improve their quality of life. TACE is a standard minimally invasive procedure developed for HCC patients who are not eligible for complete resection [4]. TACE involves the injection of a chemotherapeutic agent, which induces selective vascular embolization and blocks the arteries, hence triggering tumor infarction and necrosis [5-6] and the combination of fluorouracil, cisplatin, mitomycin or epirubicin in the TACE treatment was the most common regimen [7].Patients with large and multiple lesions exceeding the Milan criteria have been widely treated by TACE, which has been proven to improve the survival of those patients [8-10]. However, TACE may further affect liver functions and damage the hepatic arterial system. As a result, TACE is not appropriate for patients with poor liver functions, particularly those with cirrhosis, which are the limitations of TACE [11].

However, it is usually difficult to achieve complete necrosis of the target lesion by TACE alone because of the intracapsular or extracapsular invasion of unresectable $\mathrm{HCC}$ and viable tumor cells remaining after treatment [12]. Thus, repeated procedures are needed to achieve better results, although not much survival benefit has been gained with TACE alone [13-14]. Moreover, threedimensional conformal radiotherapy (3DCRT) [15], radiofrequency ablation (RFA) [16-17], percutaneous ethanol injection (PEI) [18], percutaneous acetic acid injection (PAI) [19], percutaneous microwave coagulation therapy (PMCT) [20], drug-eluting bead transarterial chemoembolization (DEB-TACE) [21], and sorafenib [22] have also been shown to be highly effective in the treatment of HCC. Studies have also suggested that TACE combined with other percutaneous techniques and targeted therapy drugs may improve survival [23-24].

Although this combination therapy has been used in patients suffering from unresectable HCC, the current data on therapeutic effects are controversial, and its clinical role has not been decided. To further explore these issues and to identify the best combination treatment with TACE, we performed a network meta-analysis of all available clinical trials of patients with unresectable HCC. No previous reviews [18, 25-27] have provided a comprehensive overview with meta-regressions and network meta-analyses.

\section{RESULTS}

\section{Description of the network and patients}

In total, 1389 citations were retrieved from the databases; after removing duplicates, 723 citations were screened on title and abstract; 587 were excluded from further analysis. A total of 123 citations were included for full-text analysis. The network consists of 48 trials and 5627 patients, which were included in the standard meta-analysis. Because of a factorial design in two trials, these 48 trials were split into 50 analyses. There were 19 different treatments: (1) TACE, (2) DEB-TACE; (3) TACE + sorafenib; (4) TACE + stereotactic body radiation therapy (TACE + SBRT); (5) TACE + TACE + PMCT; (6) TAI; (7) TACE + Licartin; (8) selective internal radiotherapy (SIRT); (9) TACE + RFA); (10) RFA; (11) TACE + brivanib; (12) TACE + 3DCRT; (13) TACE + amiodarone; (14) TACE + interferon-a (TACE + IFN); (15) TACE + PEI; (16) TACE + radiotherapy therapy (TACE + RT); (17) TACE + PAI; (18) percutaneous acetic acid injection (PAI); (19) high-dose hepatic arterial infusion chemotherapy (HAIC). Figure 1 shows the screening flowchart.

Table 1 summarizes the differences in the fundamental characteristics between the two treatment arms (see full characteristics information in Supplementary Table 1). The statistics showed that the two groups had similar baseline results in age, gender, tumor stage, tumor size, Child-Pugh, ECOG status, HBV infection rate, HCV infection rate, and number of tumors. The assessments of study quality are presented in Supplementary Table 2, and the NOS scale score result shows that all included studies had an acceptable quality.

Figure 2 displays the network weight of eligible comparisons for treatment response (A), 1-year OS (B), 2-year OS (C), and 1-year DFS (D) displaying the available direct comparisons and network of the trials. Table 2 summarizes the numbers of patients with unresectable $\mathrm{HCC}$ according to study treatment. Patients were grouped by different treatment arms (most trials only had two arms). More than half of our included trials compared the efficacy and safety of TACE plus other treatment with TACE alone.

\section{Primary efficacy outcome - Treatment response}

The network meta-analysis suggested that, in comparison with TACE treatment alone, DCB-TACE ranked the lowest for efficacy (OR: $0.41,95 \%$ CI: 0.09 1.90); TACE + PEI ranked the best for the efficacy of treatment response $(6.72,1.43-31.60)$, followed by TACE + sorafenib $(5.42,2.60-11.30)$, TACE + 3DCRT (3.10, 2.04-4.72), TACE + PMCT $(3.00,-1.11-8.12)$, HAIC $(15.13,0.00-6.72 \mathrm{e}+6)$, TAI $(15.13,0.00-6.72 \mathrm{e}+6)$, and TACE + amiodarone (1.67, 0.26-10.91). 
Table 1: Characteristics of baseline in patients associated with Treatment arm I vs Treatment arm II

\begin{tabular}{lll}
\hline & Treatment arm I vs II (OR, 95\%CI) & Heterogeneity \\
\hline Age (year) & $0.015(-0.08,0.11)^{*}$ & $P=0.890, I^{2}=0.0 \%$ \\
Male & $0.98(0.84,1.15)$ & $P=0.417, I^{2}=3.0 \%$ \\
Tumor stage (I-II/III-IV) & $0.90(0.61,1.33)$ & $P=0.210, I^{2}=23.8 \%$ \\
Tumor size (cm) (< 5/5-/< 10/10-) & $1.01(0.83,1.23)$ & $P=0.903, I^{2}=0.0 \%$ \\
Child-Pugh (A/B-C) & $0.99(0.78,1.26)$ & $P=0.063, I^{2}=31.7 \%$ \\
ECOG status (0/1) & $1.30(0.95,1.78)$ & $P=0.068, I^{2}=45.1 \%$ \\
HBV (+/-) & $0.97(0.81,1.15)$ & $P=0.971, I^{2}=0.0 \%$ \\
HCV (+/-) & $0.95(0.78,1.18)$ & $P=0.999, I^{2}=0.0 \%$ \\
Number of tumors (single/multiple) & $0.72(0.49,1.06)$ & $P=0.000, I^{2}=70.2 \%$ \\
\hline
\end{tabular}

*Standardized mean difference;

When we assessed the comparative efficacy, TACE + PEI was superior to all other treatment arms. However, the other treatment arms did not reach significance. Treatment arms were comparable with each other for improved treatment responses, with no significant differences found (Figure 3). The comparison-adjusted funnel plot of treatment response was not suggestive of any publication bias (Supplementary Figure 1).

\section{Primary efficacy outcome - OS}

\section{6-month OS}

The network meta-analysis suggested that, compared with TACE treatment alone, TACE+3DCRT was ranked best for improving 6-month OS $(4.95,1.53-$ 15.96), followed by TACE + IFN $(2.49,1.04-5.99)$, TACE + Licartin $(1.89,1.20-2.97)$, TACE + PMCT $(3.22,0.66$ -15.76), HAIC (2.33, 0.86-6.29), TACE + RFA (2.03, $0.47-8.74)$, TACE + sorafenib $(1.76,0.00-8.08 \mathrm{e}+5)$, SIRT $(1.76,0.00-8.08 \mathrm{e}+5)$, TACE + amiodarone $(1.75,0.00$ $7.75 \mathrm{e}+5)$, DEB-TACE $(1.75,0.00-7.75 \mathrm{e}+5)$, TACE + PEI (1.64, 0.48-5.61), TACE + brivanib $(1.04,0.70-1.55)$, RFA (0.89, 0.20-2.98), and TAI (0.01, 0.00-0.04).

When we assessed the comparative efficacy, TACE +3 DCRT was superior to all other treatment arms. However, except for the TACE + 3DCRT arm, TACE + IFN arm, and TACE + Licartin arm, the other treatment arms did not reach significance. Treatment arms were comparable with each other for improving 6-month OS,

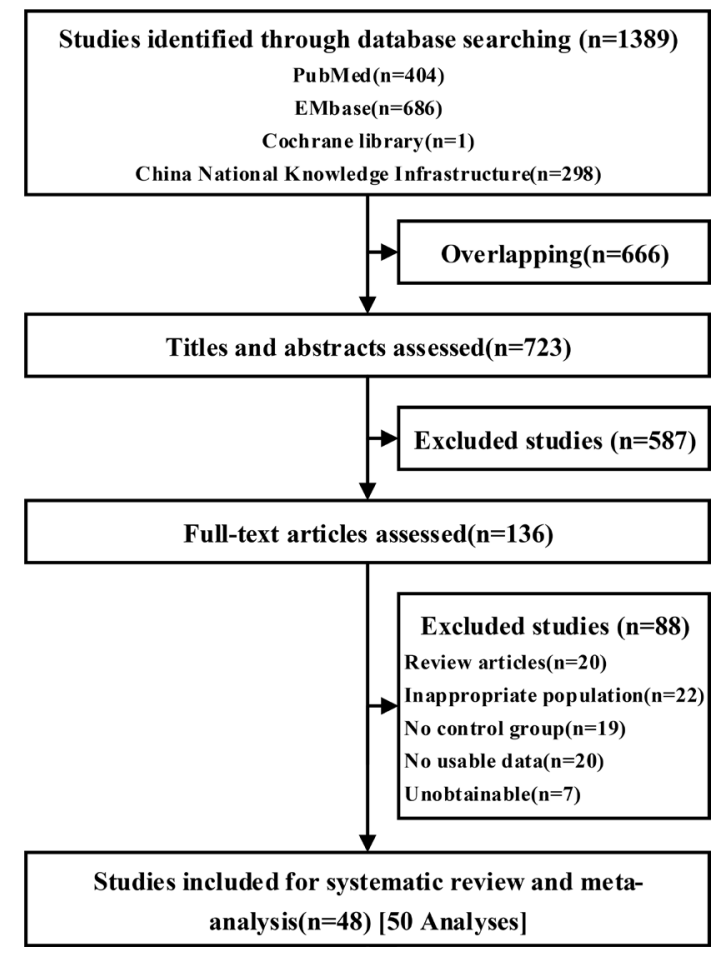

Figure 1: Flow of studies through the review process for the systematic review and network meta-analysis. 
with a significant difference found in the TACE + 3DCRT vs TAI group (404.43, 76.24-2.1e+3), TACE + 3DCRT vs TACE + brivanib group $(4.75,1.38-16.36)$, and HAIC vs TAI group (190.35, 40.43-9e+2) (Figure 4).

\section{1-year OS}

The network meta-analysis suggested that, compared with TACE treatment alone, TACE + 3DCRT was ranked best for improving 1-year OS $(2.88,2.21-3.74)$, followed by HAIC (2.98, 0.96-9.30), TACE + amiodarone (2.57, 0.48-13.60), TACE + RFA (1.61, 0.29-9.10), TACE + PAI $(7.85,0.00-1.59 \mathrm{e}+5)$, PAI $(7.85,0.00-1.59 \mathrm{e}+5), \mathrm{TACE}+$ PMCT (1.96, 1.01-3.82), TACE + RT (1.76, 0.79-3.96), TACE + sorafenib $(1.53,1.07-2.17)$, SIRT $(1.48,0.89$ 2.46), TACE + IFN (1.57, 0.- 3.34), TACE + Licartin (1.48, 0.89-2.46), TACE + PEI (1.42, 0.57-3.53), TACE + brivanib (0.74, 0.18-2.98), DEB-TACE (1.09, 0.66-1.81), TAI (1.04, 0.55-1.97), and RFA (2.32,0.59-9.05).

When we assessed the comparative efficacy, TACE + 3DCRT was superior to all other treatment arms. However, except for the TACE + 3DCRT arm, TACE+PMCT arm, and TACE + sorafenib arm, the other treatment arms did not reach significance. Treatment arms were comparable with each other for improving 1-year OS, with a significant difference found in the TACE + 3DCRT vs TACE + sorafenib group (1.88, 1.22-2.91), TACE + 3DCRT vs TACE + Licartin group (1.95, 1.10 2.24), TACE + 3DCRT vs TACE + brivanib group (2.37, 1.41-4.00), TACE + 3DCRT vs DEB-TACE group (2.65, $1.50-4.67)$, and TACE + 3DCRT vs TAI group (2.76, 1.38-5.52) (Figure 4).

Figure 5 summarizes the results of the standardized meta-analysis for 1-year OS. Overall, the TACE + 3DCRT vs TACE group was associated with a significant increase in 1-year OS $(2.87,2.24-3.69)$ with no heterogeneity $(P=$ $0.000, I^{2}=0.0 \%$ ), and the TACE + sorafenib vs TACE group was associated with an increase tendency in 1-year OS $(1.64,0.96-2.79)$ with substantial heterogeneity $(P=$ $\left.0.034, I^{2}=61.7 \%\right)$. A similar tendency could also be found in the TACE + PEI vs TACE group (2.20, 0.63-7.69; $P$ $\left.=0.156, I^{2}=42.6 \%\right)$, DEB-TACE vs TACE group (1.06, $\left.0.67-1.68 ; P=0.440, P^{2}=0.0 \%\right)$, TACE + PMCT vs TACE group (2.00, 0.90-4.44; $P=0.215, I^{2}=35.1 \%$ ), TACE + RFA vs RFA group (2.40, 0.99-5.80; $P=0.001$, $I^{2}=73.4 \%$ ), while the opposite tendency could be found in the TACE vs TAI group $(0.95,0.53-1.73 ; P=0.431$,
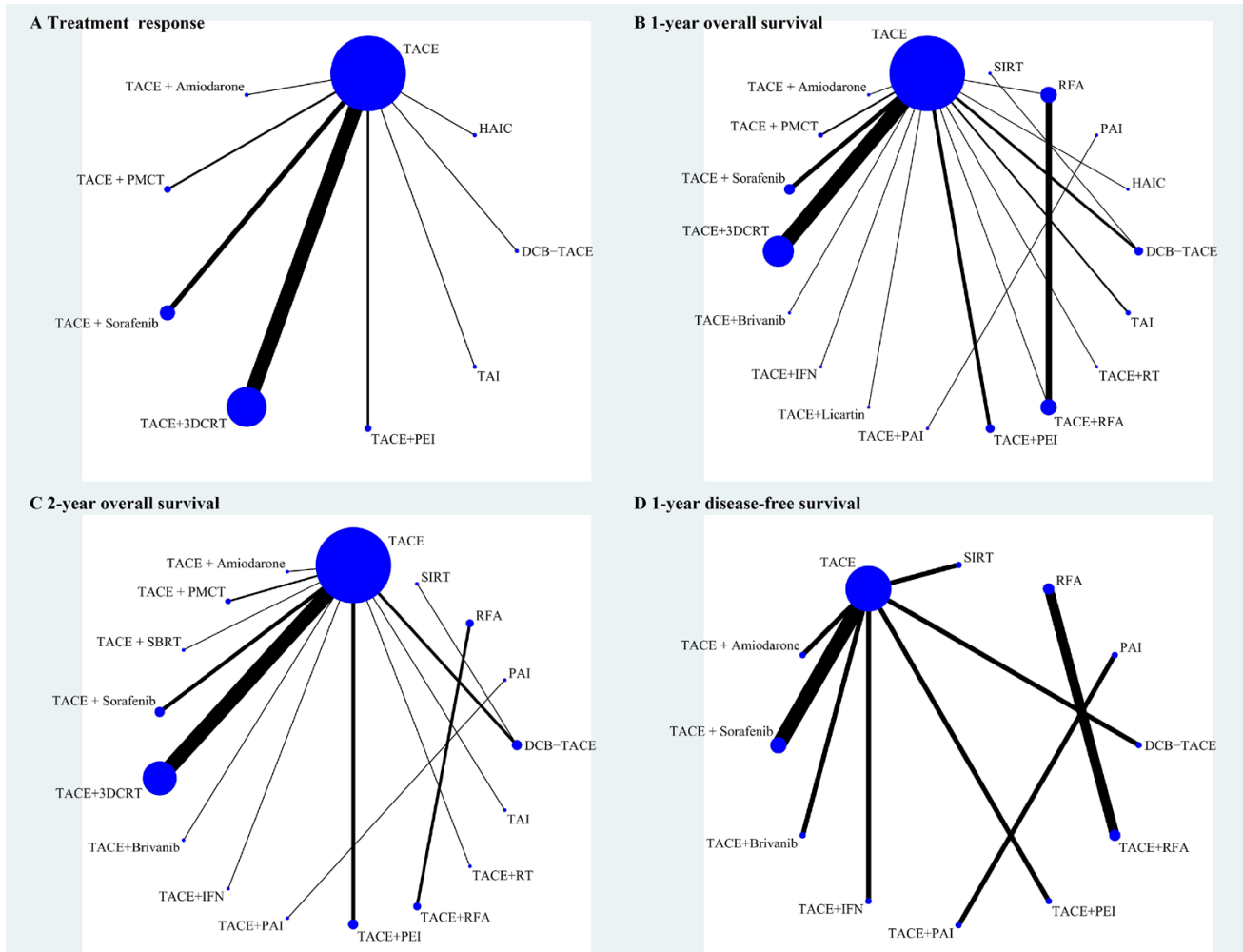

Figure 2: Network of eligible comparisons for treatment response (A), 1-year overall survival (B), 2-year overall survival (C) and 1-year disease-free survival (D). The width of the lines is proportional to the number of trials comparing every pair of treatments, and the size of every circle is proportional to the number of randomly assigned participants (sample size). Abbreviations: 3DCRT, three-dimension eonformal radiotherapy; DEB-TACE, drug-eluting bead transarterial chemoembolization; HAIC, high-dose hepatic arterial infusion chemotherapy; IFN, interferon-a; PAI, percutaneous acetic acid injection; PEI, percutaneous ethanol injection; PMCT, percutaneous microwave coagulation therapy; RFA, radiofrequency ablation; RT, radiotherapy therapy; SBRT, stereotactic body radiation therapy; SIRT, selective internal radiotherapy; TACE, transarterial chemoembolization; TAI, transarterial infusion chemotherapy. 
Table 2: Number of patients with unresectable hepatocellular carcinoma according to study treatment

\begin{tabular}{llll}
\hline Treatment arm I & Treatment arm II & Number of analyses & Patients(I/II) \\
\hline TACE + 3DCRT & TACE & $16[38,42,44-45,49-50,55,57-58,62-64,66-69]$ & $615 / 645$ \\
TACE + Sorafenib & TACE & $6[29,39,41,48,51,53]$ & $528 / 660$ \\
DCB-TACE & TACE & $3[28,31,52]$ & $154 / 239$ \\
TACE + PMCT & TACE & $2[32,46]$ & $102 / 116$ \\
TACE+Licartin & TACE & $1[34]$ & $167 / 174$ \\
TACE+Brivanib & TACE & $1[37]$ & $249 / 253$ \\
TACE + Amiodarone & TACE & $1[47]$ & $13 / 14$ \\
TACE+IFN & TACE & $1[59]$ & $108 / 108$ \\
TACE+RFA & TACE & $1[65]$ & $24 / 11$ \\
TACE+PEI & TACE & $4[70,73-75]$ & $102 / 100$ \\
TACE+RT & TACE & $1[71]$ & $54 / 60$ \\
TACE + SBRT & TACE & $1[30]$ & $44 / 52$ \\
TACE + PAI & PAI & $1[72]$ & $53 / 55$ \\
TACE & TAI & $2[33,60]$ & $178 / 177$ \\
DCB-TACE & SIRT & $1[35]$ & $12 / 12$ \\
TACE+RFA & RFA & $7[36,40,43,56,61,65]$ & $270 / 235$ \\
HAIC & TACE & $1[54]$ & $31 / 36$ \\
\hline
\end{tabular}

3DCRT, three-dimension eonformal radiotherapy; DEB-TACE, drug-eluting bead transarterial chemoembolization; HAIC, high-dose hepatic arterial infusion chemotherapy; IFN, interferon-a; PAI, percutaneous acetic acid injection; PEI, percutaneous ethanol injection; PMCT, percutaneous microwave coagulation therapy; RFA, radiofrequency ablation; RT, radiotherapy therapy; SBRT, stereotactic body radiation therapy; SIRT, selective internal radiotherapy; TACE, transarterial chemoembolization; TAI, transarterial infusion chemotherapy.

$\left.I^{2}=0.0 \%\right)$. The network and standardized meta-analysis results both revealed that TACE + 3DCRT has a significant, positive effect. The comparison-adjusted funnel plot of 1 -year OS was not suggestive of any publication bias (Supplementary Figure 2). What's more, some evidence of bias could be found in Begg's $(P=0.020)$ and Egger's $(P=0.000)$ tests, with moderate quality evidence according to the GRADE assessment (Supplementary Table 3).

\section{2-year OS}

The network meta-analysis suggested that, compared with TACE treatment alone, TACE+PAI was ranked best for improving 2-year OS $(21.74,0.02-2.21 \mathrm{e}+3)$, followed by TACE+PMCT $(3.65,1.83-7.31)$, TACE + RFA (12.73, $0.02-8.16 \mathrm{e}+3), \mathrm{TACE}+$ sorafenib $(3.10,1.77-5.45)$, TACE + 3DCRT $(2.92,2.28-3.73)$,TACE + PEI (2.97, 1.46-6.02), RFA (9.46, 0.01-6.05e+3), PAI (9.16, 0.01$9.27 \mathrm{e}+3)$, TACE + IFN $(2.22,1.27-3.88)$, TACE + SBRT (1.73, 0.74-4.04), TACE + amiodarone (1.65, 0.34-8.08), TACE + RT (1.62, 0.77-3.43), DEB-TACE $(1.28,0.80$ $2.03)$, SIRT $(0.96,0.18-5.25)$, TAI $(1.10,0.59-2.06)$, and TACE + brivanib $(0.85,0.47-1.55)$.

When we assessed the comparative efficacy, TACE + PAI was superior to all other treatment arms. However, except for the TACE+PMCT arm, TACE + sorafenib arm,
TACE + 3DCRT arm, TACE + PEI arm, and TACE + IFN arm, the other treatment arms did not reach significance. Treatment arms were comparable with each other for improving 2-year OS, with significant difference found in the TACE + sorafenib vs TACE + brivanib group (3.57, 1.61-8.33), TACE + 3DCRT vs TACE + brivanib group (3.42, 1.79-6.51), TACE + IFN vs TACE + brivanib group $(3.47,1.38-8.77)$, TACE + amiodarone vs TACE + brivanib group $(2.60,1.15-5.88)$, TAI vs TACE + brivanib group $(2.65,1.35-5.17)$, TACE+PMCT vs TAI (3.84, 0.61-25.00), TACE + sorafenib vs TAI $(2.78,1.22-6.67)$, TACE + IFN vs TAI $(2.69,1.05-6.91)$, TACE + sorafenib vs DEB-TACE $(2.43,1.17-5.05)$, TACE + 3DCRT vs DEB-TACE $(2.29,1.35-3.87)$, TACE + PEI vs DEBTACE $(2.32,1.00-5.42)$, PAI vs TACE + SBRT (3.33, 1.30-8.33), and TACE + PMCT vs RFA (4.35, 1.72-11.11) (Figure 6).

\section{3-year OS}

The network meta-analysis suggested that, compared with TACE treatment alone, TACE + PAI was ranked best for improving 3-year OS (47.16, 0.01-1.57e+5), followed by TACE + RFA (7.72, 0.01-6731.08), RFA (4.66, 0.014058.47), PAI (4.34, 0.00-1.44e+4), TACE + 3DCRT $(3.35,2.41-4.66)$, TACE + PEI $(2.84,1.21-6.63)$, TACE 
+ RT (1.13, 2.28-6.21), TACE + amiodarone (2.51, $0.21-29.81)$, TACE + IFN $(2.13,1.24$ to 3.66), TAI (0.96, 0.46-2.02), DEB-TACE $(0.77,0.43-1.39)$, and TACE + brivanib (32.77, 0.00-7.58e+6).

When we assessed the comparative efficacy, TACE + PAI was superior to all other treatment arms. However, except for the TACE + 3DCRT arm, TACE + PEI arm, TACE + IFN arm, and TACE + RT arm, the other treatment arms did not reach significance. Treatment arms were comparable with each other for improving 3-year OS, with significant differences found in the TACE + 3DCRT vs TAI group $(3.48,1.55-7.82)$, TACE + 3DCRT

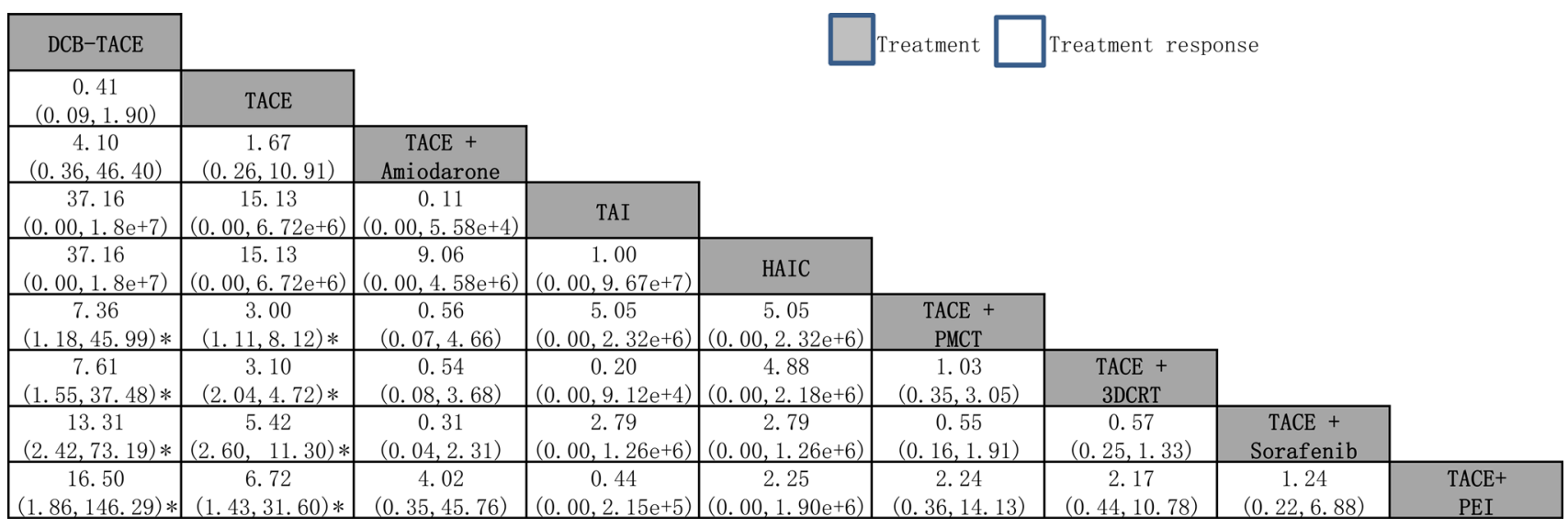

Figure 3: Network meta-analysis of treatment response. Treatments are reported in order of survival rate ranking according to SUCRA of treatment response. Comparisons should be read from left to right. The estimate is located at the intersection of the columndefining treatment and the row-defining treatment. For treatment response, an OR value above 1 favors the treatment arm. "Result with significant difference. Abbreviations: DEB-TACE, drug-eluting bead transarterial chemoembolization; HAIC, high-dose hepatic arterial infusion chemotherapy; PEI, percutaneous ethanol injection; PMCT, percutaneous microwave coagulation therapy; TACE, transarterial chemoembolization; TAI, transarterial infusion chemotherapy.

\begin{tabular}{|c|c|c|c|c|c|c|c|c|c|c|c|c|c|c|c|c|c|}
\hline TACE & $\begin{array}{c}.89 \\
(0.20,3.98) \\
\end{array}$ & $\begin{array}{c}0.01 \\
(0.00,0.04) \\
0.505\end{array}$ & $\begin{array}{c}1.75 \\
(0.00,7.75 \mathrm{e}+5) \\
\end{array}$ & \begin{tabular}{c|c}
1.04 \\
$(0.70,1.55)$ \\
\end{tabular} & \begin{tabular}{c|}
1.64 \\
$(0.48,5.61)$ \\
\end{tabular} & $\begin{array}{c}1.89 \\
(1.22,2.97)^{*} \\
\end{array}$ & $\begin{array}{c}2.49 \\
(1.04,599)^{*} \\
\end{array}$ & \begin{tabular}{c|c}
1.76 \\
$(0.00,8.88 \mathrm{e}+5)$ \\
\end{tabular} & \begin{tabular}{c|}
1.76 \\
$(0.00,8.08 \mathrm{e}+5)$ \\
\end{tabular} & - & \begin{tabular}{|c|}
3.22 \\
$(0.66,15.76)$ \\
\end{tabular} & & - & \begin{tabular}{c|c|}
2.03 \\
$(0.47,8,74)$ \\
\end{tabular} & $\begin{array}{cc}1 & 1.75 \\
(0.00,7.75 \mathrm{e}+5) \\
\end{array}$ & \begin{tabular}{c|c|}
2.33 \\
$(0.86,6.29)$ \\
\end{tabular} & \begin{tabular}{c|c|}
4.95 \\
$(1.53,15.96)^{*}$ \\
\end{tabular} \\
\hline $\begin{array}{c}2.32 \\
(0.59,9.05)\end{array}$ & RFA & \begin{tabular}{|c|c}
72.50 \\
$(10.71,4.9 \mathrm{e}+2)$ \\
(
\end{tabular} & $\begin{array}{c}0.51 \\
(0.00,2.40 \mathrm{e}+4) \\
\end{array}$ & $\begin{array}{c}1.17 \\
(0.25,5.54) \\
\end{array}$ & \begin{tabular}{|c|c}
1.85 \\
$(0.27,12.84)$ \\
\end{tabular} & $\begin{array}{c}0.47 \\
(0.10,2.25) \\
\end{array}$ & $\begin{array}{c}2.81 \\
(0.50,15.96) \\
\end{array}$ & $\begin{array}{c}0.50 \\
(0.00,2.47 \mathrm{e}+4)\end{array}$ & \begin{tabular}{|c|c|c|}
0.50 \\
$(0.0,2,247 \mathrm{e}+4)$ \\
\end{tabular} & - & \begin{tabular}{|c|}
0.28 \\
$(0.03,2,44)$ \\
\end{tabular} & & - & \begin{tabular}{c|c}
0.44 \\
$(0.18,1.07)$ \\
\end{tabular} & \begin{tabular}{|c|}
1.98 \\
$\left(0.00,9,377^{+}+4\right)$ \\
\end{tabular} & $\begin{array}{c}2.63 \\
(0.44,15.84) \\
\end{array}$ & $\begin{array}{c}5.58 \\
(0.83,37.71) \\
\end{array}$ \\
\hline $\begin{array}{c}1.04 \\
(0.55,197)\end{array}$ & $\begin{array}{c}0.71 \\
(0.15,3.29) \\
\end{array}$ & TAI & $\begin{array}{c}0.01 \\
(0.00,326.01) \\
\end{array}$ & \begin{tabular}{|c|}
85.10 \\
$(24,28,29+2+2)$
\end{tabular} & $\begin{array}{c}134,36 \\
(24,32,7 \cdot 4+2+2) \\
\end{array}$ & $\begin{array}{c}154.23 \\
(43.23,55.104) \\
\end{array}$ & $\begin{array}{c}203.80 \\
(46.49,89+9+2) \\
\end{array}$ & $\begin{array}{c}144.28 \\
(0.00,6.99 \mathrm{e}-6)\end{array}$ & \begin{tabular}{|c|c|}
0.01 \\
$(0.00,335.76)$ \\
\end{tabular} & - & \begin{tabular}{|c|c}
0.00 \\
$(0.00,0.03)$ \\
\end{tabular} & - & - & \begin{tabular}{|l|}
$1255.757 .1 \mathrm{le}+3)$ \\
\end{tabular} & \begin{tabular}{|c|c|}
143.37 \\
$(0.00,677+6)$ \\
\end{tabular} & $\begin{array}{c}190.35 \\
(40.43,9 \mathrm{e}+2)^{*} \\
\end{array}$ & 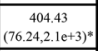 \\
\hline $\begin{array}{c}109 \\
(0.66,1.81)\end{array}$ & $\begin{array}{c}0.68 \\
(0.15,2.99) \\
\end{array}$ & $\begin{array}{c}0.96 \\
(0.42,218) \\
\end{array}$ & DCB-TACE & $\begin{array}{c}0.59 \\
(0.00,2.6 \mathrm{ec}+4) \\
\end{array}$ & $\begin{array}{c}0.94 \\
(0.00,439 \mathrm{e}+4) \\
\end{array}$ & $\begin{array}{c}1.08 \\
(0.00,499+4) \\
\end{array}$ & $\begin{array}{c}1.42 \\
(0.00,648 \mathrm{e}+4) \\
\end{array}$ & $\begin{array}{c}1.01 \\
(0.002 .24 \mathrm{e}+6)\end{array}$ & $\begin{array}{c}1.01 \\
(0.00,75 \mathrm{se}+4) \\
\end{array}$ & - & \begin{tabular}{|c|}
1.83 \\
$(0.00,897 \mathrm{c}+4)$ \\
\end{tabular} & & - & \begin{tabular}{c|c}
1.16 \\
$(0.00,546 \mathrm{e}-4)$ \\
\end{tabular} & $\begin{array}{c}1.00 \\
(0.00,2.05 \mathrm{e}+6)\end{array}$ & $\begin{array}{c}1.33 \\
(0.00,923 \mathrm{e}-4) \\
\end{array}$ & $\begin{array}{c}2.82 \\
(0.00,1.32+5) \\
\end{array}$ \\
\hline $\begin{array}{c}0.74 \\
(0.182 .98)\end{array}$ & $\begin{array}{c}1.64 \\
(0.387 .09) \\
\end{array}$ & $\begin{array}{c}1.16 \\
(0.53 .2 .53) \\
\end{array}$ & $\begin{array}{c}1.12 \\
(0.57 .220) \\
\end{array}$ & $\begin{array}{c}\text { TACE } \\
\text { +Brivanib } \\
\end{array}$ & $\begin{array}{c}1.58 \\
(0.43 .574) \\
\end{array}$ & $\begin{array}{c}0.55 \\
(0.201 .101) \\
\end{array}$ & $\begin{array}{c}2.39 \\
(0.91,6.27) \\
\end{array}$ & \begin{tabular}{|c|}
0.59 \\
$(0.00,2.72 \mathrm{e}+4)$ \\
\end{tabular} & \begin{tabular}{|c|}
0.59 \\
$(0.00 .2 .720+4)$ \\
\end{tabular} & - & \begin{tabular}{cc|}
0.32 \\
$(0.06,1.66)$ \\
\end{tabular} & - & - & \begin{tabular}{|c|}
0.51 \\
$(0.11,2,33)$ \\
\end{tabular} & $\begin{array}{c}1.68 \\
(0.007,799+4) \\
\end{array}$ & \begin{tabular}{c|}
2.24 \\
$(0.77,6.51)$ \\
\end{tabular} & $\begin{array}{c}4.75 \\
(1.38,16.3)^{*} \\
\end{array}$ \\
\hline $\begin{array}{c}1.42 \\
(0.57 .353)\end{array}$ & $\begin{array}{c}1.92 \\
(0.36,10.12) \\
\end{array}$ & $\begin{array}{c}1.36 \\
(0.45,4.16) \\
\end{array}$ & $\begin{array}{c}1.31 \\
(0.46 .3 .99) \\
\end{array}$ & $\begin{array}{c}1.17 \\
(0.42,3.24) \\
\end{array}$ & $\begin{array}{c}\begin{array}{c}\text { TACEE } \\
\text { +PEI }\end{array} \\
\end{array}$ & $\begin{array}{c}1.23 \\
(0.413 .67) \\
\end{array}$ & $\begin{array}{c}0.66 \\
(0.15,2,98) \\
\end{array}$ & $\begin{array}{c}0.93 \\
(0.00,4.42+4) \\
\end{array}$ & \begin{tabular}{|c|}
0.93 \\
$(0.00,4.520+4)$ \\
\end{tabular} & - & \begin{tabular}{|c}
0.51 \\
$(0.07,3.80)$ \\
\end{tabular} & - & - & \begin{tabular}{|c|}
1.15 \\
$(0.20,670)$ \\
\end{tabular} & $\begin{array}{c}0.94 \\
(0.00,4.39+4) \\
(0.4)\end{array}$ & $\begin{array}{c}1.42 \\
(0.29,6.87) \\
\end{array}$ & $\begin{array}{c}0.33 \\
(0.06,1.81) \\
\end{array}$ \\
\hline $\begin{array}{c}1.48 \\
(0.89,2.46)\end{array}$ & $\begin{array}{c}0.50 \\
(0.11,2.21) \\
\end{array}$ & $\begin{array}{c}1.42 \\
(0.62,32.11) \\
\end{array}$ & $\begin{array}{c}1.36 \\
(0.66,2.79) \\
\end{array}$ & $\begin{array}{c}0.82 \\
(0.42,1.62) \\
\end{array}$ & $\begin{array}{c}0.96 \\
(0.34,2.74)\end{array}$ & $\begin{array}{l}\text { TACE } \\
\text { +Licartin } \\
\end{array}$ & $\begin{array}{c}0.87 \\
(0.24,3.22) \\
\end{array}$ & $\begin{array}{c}0.93 \\
10.00 .41 .14 \mathrm{e}\end{array}$ & \begin{tabular}{|c|c}
1.07 \\
$(0.00,4,94+4+4)$ \\
\end{tabular} & - & \begin{tabular}{|c|}
0.59 \\
$(0.11,3.06)$ \\
\end{tabular} & - & . & \begin{tabular}{c|c}
1.07 \\
$(0.23,495)$ \\
\end{tabular} & $\begin{array}{c}0.93 \\
(0.00,4.140+4) \\
\end{array}$ & $\begin{array}{c}1.23 \\
(0.41,3.67)\end{array}$ & $\begin{array}{c}2.62 \\
(0.75,9.19) \\
\end{array}$ \\
\hline $\begin{array}{c}1.57 \\
(0.74,3.34) \\
\end{array}$ & $\begin{array}{c}2.12 \\
(0.44,10.34) \\
\end{array}$ & $\begin{array}{c}1.51 \\
(0.56,4.05) \\
\end{array}$ & $\begin{array}{c}1.44 \\
(0.58,3.58) \\
\end{array}$ & $\begin{array}{c}1.29 \\
(0.54,3.12) \\
\end{array}$ & $\begin{array}{c}1.24 \\
(0.37,4.20) \\
\end{array}$ & $\begin{array}{c}1.06 \\
(0.43,2.65) \\
\end{array}$ & $\begin{array}{l}\text { TACE } \\
\text { tIFN }\end{array}$ & $\begin{array}{c}1.41 \\
(0.00,6.67 \mathrm{et}+ \\
\end{array}$ & $\begin{array}{c}1.41 \\
(0.00,6.677+4) \\
\end{array}$ &. & \begin{tabular}{|c|}
0.77 \\
$(0.13,4.75)$ \\
\end{tabular} & - & . & \begin{tabular}{|c|}
1.23 \\
$(0.22,6.75)$ \\
\end{tabular} & $\begin{array}{c}1.42 \\
(0.00,6.47 \mathrm{e}+4)\end{array}$ & $\begin{array}{c}0.93 \\
(0.25,3.51)\end{array}$ & $\begin{array}{c}0.50 \\
(0.12,2,18) \\
\end{array}$ \\
\hline $\begin{array}{c}1.48 \\
(0.89,240) \\
\end{array}$ & $\begin{array}{c}0.46 \\
(0.05,4,21) \\
\end{array}$ & $\begin{array}{c}1.55 \\
(0.24,982) \\
\end{array}$ & $\begin{array}{c}1.48 \\
(0.28,780) \\
\end{array}$ & \begin{tabular}{c|}
0.75 \\
$(0.13,450)$ \\
\end{tabular} & $\begin{array}{c}0.88 \\
(0.12,621) \\
\end{array}$ & $\begin{array}{c}1.09 \\
(0.18,6.64) \\
\end{array}$ & $\begin{array}{c}0.97 \\
(0.15,643) \\
\end{array}$ & SIRT & \begin{tabular}{|c|}
1.00 \\
$(0.00,2,47 \mathrm{r}+6)$ \\
\end{tabular} & - & $\begin{array}{c}0.55 \\
(0.00,2.78 \mathrm{e}+4) \\
\end{array}$ & - & . & $\left.\begin{array}{c}1.15 \\
(0.00,5.52 e+4)\end{array}\right)$ & $\begin{array}{c}0.99 \\
(0.00,2.38 \mathrm{e}+6) \\
\end{array}$ & $\left.\begin{array}{|c|c}1.32 \\
(0.00,6,28 \mathrm{e}+4)\end{array}\right)$ & $\begin{array}{r}2.80 \\
0.00,1.35\end{array}$ \\
\hline $\begin{array}{c}1.53 \\
(1.07,2.17)^{*}\end{array}$ & $\begin{array}{c}0.48 \\
(0.12,2.03) \\
\end{array}$ & $\begin{array}{c}0.68 \\
(0.33,1.42) \\
\end{array}$ & $\begin{array}{c}1.41 \\
(0.77,258) \\
\end{array}$ & $\begin{array}{c}0.79 \\
(0.41,141) \\
\end{array}$ & \begin{tabular}{c|c}
0.93 \\
$(0.352 .46)$ \\
\end{tabular} & $\begin{array}{c}0.97 \\
(0.52,1.80)\end{array}$ & $\begin{array}{c}1.03 \\
(0.45,2.36) \\
\end{array}$ & \begin{tabular}{|c|c}
1.06 \\
$(0.18,615)$ \\
\end{tabular} & 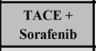 & . & \begin{tabular}{|c|}
1.82 \\
$(0.00,923 \mathrm{e}+4)$ \\
\end{tabular} & . & - & $\begin{array}{c}1.15 \\
(0.00,5,52 \mathrm{e}-4) \\
\end{array}$ & \begin{tabular}{|c|c|}
0.99 \\
$(0.00,2.38 \mathrm{e} 6)$ \\
\end{tabular} & $\begin{array}{c}1.32 \\
0.00,628 \mathrm{e}+4) \\
\end{array}$ & $\begin{aligned} 2.80 \\
0.00,135 .\end{aligned}$ \\
\hline $\begin{array}{c}1.76 \\
(0.79,396)\end{array}$ & $\begin{array}{c}2.38 \\
(0.48,11.92)\end{array}$ & $\begin{array}{c}1.69 \\
(0.60,475)\end{array}$ & $\begin{array}{c}1.62 \\
(0.63,4.21)\end{array}$ & $\begin{array}{c}1.45 \\
(0.58,3.67) \\
\end{array}$ & \begin{tabular}{c|c}
0.55 \\
$(0.083 .370)$
\end{tabular} & $\begin{array}{c}1.19 \\
(0.46,3.11)\end{array}$ & $\begin{array}{c}1.24 \\
(0.37,4.20)\end{array}$ & $\begin{array}{c}1.09 \\
(0.16,738) \\
\end{array}$ & $\begin{array}{c}1.15 \\
(0.8,2.79)\end{array}$ & $\begin{array}{c}\text { TACE } \\
\text { +RT }\end{array}$ & . & - & - &. & - & - & . \\
\hline $\begin{array}{c}196 \\
(1.01,3.82)^{*}\end{array}$ & $\begin{array}{c}0.38 \\
(0.081 .77)\end{array}$ & $\begin{array}{c}0.53 \\
(0.211 .134)\end{array}$ & $\begin{array}{c}1.80 \\
(0.78 .415)\end{array}$ & $\begin{array}{c}0.62 \\
(0.28,1.38)\end{array}$ & $\begin{array}{c}0.72 \\
(0.232 .23) \\
\end{array}$ & $\begin{array}{c}0.75 \\
(0.33,1.74)\end{array}$ & $\begin{array}{c}0.80 \\
(0.292 .19)\end{array}$ & \begin{tabular}{|c|}
.082 \\
$(0.135 .24)$ \\
\end{tabular} & $\begin{array}{c}1.28 \\
(0.61,2.72)\end{array}$ & $\begin{array}{c}0.90 \\
(0.32 .256)\end{array}$ & $\begin{array}{c}\begin{array}{c}\text { TACE } \\
+ \text { PMCT }\end{array} \\
\end{array}$ & - & - & $\begin{array}{c}0.63 \\
(0.07,543) \\
\end{array}$ & $\begin{array}{c}0.54 \\
(0.00,2.660+4)\end{array}$ & $\begin{array}{c}0.72 \\
(0.11,4.71)\end{array}$ & $\begin{array}{c}1.54 \\
(0.21,11.05\end{array}$ \\
\hline $\begin{array}{c}7.5 \\
(0.00,1.59 \mathrm{e}-5)\end{array}$ & $\begin{array}{c}10.61 \\
(0.00 .231(+5)\end{array}$ & $\begin{array}{c}7.54 \\
(0.00 .1 .56 \mathrm{e}+4)\end{array}$ & $\begin{array}{c}7.22 \\
(0.00,1.148 \mathrm{e}-4)\end{array}$ & $\begin{array}{c}6.47 \\
(0.00,1.132 \mathrm{e}+5)\end{array}$ & $\begin{array}{c}5.00 \\
(0.00,1.104+5)\end{array}$ & $\begin{array}{c}532 \\
(0.00,1.09 \mathrm{e}+5)\end{array}$ & $\begin{array}{c}5.53 \\
(0.00,1.17 \mathrm{r}+5)\end{array}$ & $\begin{array}{c}4.87 \\
(0.00,1.140+5)\end{array}$ & $\begin{array}{c}5.14 \\
(0.00,1.05 \mathrm{e} 55)\end{array}$ & $\begin{array}{c}4.45 \\
(0.00,930 \mathrm{e}+4)\end{array}$ & \begin{tabular}{|c|}
4.00 \\
$(0.00 .828 \mathrm{e}+4)$ \\
\end{tabular} & PAI & - & - & - & - & \\
\hline $\begin{array}{c}7.5 \\
(0.00,1,159+5)\end{array}$ & $\begin{array}{c}10.61 \\
(0.00,231 e+5)\end{array}$ & $\begin{array}{c}77.54 \\
(0.00,1.56 \mathrm{e}+4)\end{array}$ & $\begin{array}{c}7.22 \\
(0.00,1.148 \mathrm{e}-4)\end{array}$ & \begin{tabular}{|c|}
6.47 \\
$(0.00,1,132+5)$
\end{tabular} & $\begin{array}{c}5.00 \\
(0.00,1.04+5)\end{array}$ & $\begin{array}{c}5.32 \\
(0.00,1.09+5)\end{array}$ & $\begin{array}{c}5.53 \\
(0.00,1.17 e+5)\end{array}$ & \begin{tabular}{|c|}
4.87 \\
$(0.00,1.14 \mathrm{e}+5)$ \\
\end{tabular} & $\begin{array}{c}5.14 \\
(0.00,1.105 \mathrm{~s}+5) \\
\end{array}$ & $\begin{array}{c}4.45 \\
(0.00,930 \mathrm{e}+4)\end{array}$ & \begin{tabular}{|c|}
4.00 \\
$(0.00,828 \mathrm{e}+4)$ \\
\end{tabular} & $\left(\begin{array}{c}1.00 \\
(0.00,1.23 \mathrm{e}+6)\end{array}\right.$ & 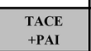 & - & - & - & - \\
\hline $\begin{array}{c}1.61 \\
(0.299 .10)\end{array}$ & $\begin{array}{c}3.13 \\
(1.72,56)^{*}\end{array}$ & $\begin{array}{c}2.23 \\
(0.49,10.02)\end{array}$ & $\begin{array}{c}2.13 \\
(0.509 .99)\end{array}$ & $\begin{array}{c}0.52 \\
(0.12,2.19)\end{array}$ & $\begin{array}{c}0.61 \\
(0.12,3.14)\end{array}$ & $\begin{array}{c}1.57 \\
(0.37,6.42)\end{array}$ & $\begin{array}{c}0.68 \\
(0.14,3.21)\end{array}$ & $\begin{array}{c}1.44 \\
(0.16,12.93)\end{array}$ & $\begin{array}{c}1.52 \\
(0.37,6.18)\end{array}$ & $\begin{array}{c}0.76 \\
(0.16,370)\end{array}$ & $\begin{array}{c}1.18 \\
(0.26 .537)\end{array}$ & $\begin{array}{c}3.39 \\
(0.00,7.34+2\end{array}$ & $\begin{array}{c}3.39 \\
(0.00,7.74 \mathrm{e}+4)\end{array}$ & 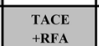 & $\begin{array}{c}0.86 \\
(0.00,4.08+4)\end{array}$ & $\begin{array}{c}1.17 \\
(0.25,5.54)\end{array}$ & $\begin{array}{c}2.44 \\
(0.38,15.82)\end{array}$ \\
\hline $\begin{array}{c}2.57 \\
(0.48,13.60)\end{array}$ & $\begin{array}{c}3.47 \\
(0.40,30.34)\end{array}$ & $\begin{array}{c}2.46 \\
(041,1469)\end{array}$ & $\begin{array}{c}2.36 \\
(0.41,13.47)\end{array}$ & $\begin{array}{c}2.11 \\
(0.38,11.90)\end{array}$ & $\begin{array}{c}0.55 \\
(0.083 .70) \\
\end{array}$ & $\begin{array}{c}1.74 \\
(030,994)\end{array}$ & $\begin{array}{c}0.61 \\
(0.10,3.82)\end{array}$ & $\begin{array}{c}1.59 \\
(0.14,1757)\end{array}$ & $\begin{array}{c}1.68 \\
(031,923) \\
\end{array}$ & $\begin{array}{c}0.69 \\
(0.11,439)\end{array}$ & $\begin{array}{c}1.31 \\
(0.227 .78) \\
\end{array}$ & $\begin{array}{c}5.00 \\
(000,1.04+5) \\
\end{array}$ & $\begin{array}{c}5.00 \\
(0.00,1.04+5)\end{array}$ & $\begin{array}{c}1.11 \\
(0.13949)\end{array}$ & $\begin{array}{c}\text { TACE + } \\
\text { Amiodarone }\end{array}$ & $\begin{array}{c}1.33 \\
(0.00,6.1 \mathrm{le}+4) \\
\end{array}$ & $\begin{array}{c}0.35 \\
(0.001 .65 \mathrm{et} 4\end{array}$ \\
\hline $\begin{array}{c}2.98 \\
(0.969 .90)\end{array}$ & $\begin{array}{c}4.03 \\
(0.67,2430)\end{array}$ & $\begin{array}{c}2.86 \\
(0.78,1056)\end{array}$ & $\begin{array}{c}2.74 \\
(0.79,953)\end{array}$ & $\begin{array}{c}2.46 \\
(0.22,836)\end{array}$ & $\begin{array}{c}1.90 \\
(0.497 .74)\end{array}$ & $\begin{array}{c}2.02 \\
(0.587 .03)\end{array}$ & $\begin{array}{c}2.10 \\
(0.49,9.03)\end{array}$ & $\begin{array}{c}1.85 \\
(028,14.66)\end{array}$ & $\begin{array}{c}1.95 \\
(0.59,642)\end{array}$ & $\begin{array}{c}1.69 \\
(0.43,6.82)\end{array}$ & $\begin{array}{c}1.52 \\
(0.41 .568)\end{array}$ & $\begin{array}{c}.38 \\
(0.00,8.19+3)\end{array}$ & $\begin{array}{c}0.38 \\
(0.00 .8 .19 \mathrm{e}+3)\end{array}$ & $\begin{array}{c}1.29 \\
(0.22,757)\end{array}$ & $\begin{array}{c}1.90 \\
(049,7.44)\end{array}$ & HAIC & $\begin{array}{c}0.47 \\
(0.10,218)\end{array}$ \\
\hline $\begin{array}{c}2.88 \\
(2.21,3.74)^{*}\end{array}$ & $\begin{array}{c}3.89 \\
(0.94,16.05)\end{array}$ & $\begin{array}{c}2.76 \\
(1.38,5.52)^{*}\end{array}$ & $\begin{array}{c}2.65 \\
(1.50,4.67)^{*}\end{array}$ & $\begin{array}{c}2.37 \\
(1.41,4.0)^{*}\end{array}$ & $\begin{array}{c}0.49 \\
(0.19,1.27)\end{array}$ & $\begin{array}{c}195 \\
(1.102 .224)^{*}\end{array}$ & $\begin{array}{c}0.55 \\
(0.25,1.21)\end{array}$ & $\begin{array}{c}1.78 \\
(0.31,1028) \\
\end{array}$ & $\begin{array}{c}1.88 \\
(1.22 .2 .91)^{2}\end{array}$ & $\begin{array}{c}0.61 \\
(0.261 .43) \\
\end{array}$ & $\begin{array}{c}1.47 \\
(0.72 .300) \\
\end{array}$ & $\begin{array}{c}2.73 \\
(0.00 .553 \mathrm{se}+4) \\
\end{array}$ & $\begin{array}{c}2.73 \\
(0.00 .55 \mathrm{~s}+4) \\
\end{array}$ & $\begin{array}{c}1.24 \\
(0.31 .496) \\
\end{array}$ & $\begin{array}{c}0.89 \\
(0.16,4.82) \\
\end{array}$ & $\begin{array}{c}104 \\
(0.32 .333) \\
\end{array}$ & $\begin{array}{l}\text { TACE } \\
+3 \text { BCRT } \\
\end{array}$ \\
\hline
\end{tabular}

Figure 4: Network meta-analysis of 6-months OS and 1-year OS. Treatments are reported in order of survival rate ranking according to SUCRA of treatment response. Comparisons should be read from left to right. The estimate is located at the intersection of the column-defining treatment and the row-defining treatment. For OS, an OR value above 1 favors the treatment arm. *Result with significant difference. Abbreviations: 3DCRT, three-dimension eonformal radiotherapy; DEB-TACE, drug-eluting bead transarterial chemoembolization; HAIC, high-dose hepatic arterial infusion chemotherapy; IFN, interferon-a; PAI, percutaneous acetic acid injection; PEI,percutaneous ethanol injection; PMCT, percutaneous microwave coagulation therapy; RFA, radiofrequency ablation; RT, radiotherapy therapy; SIRT, selective internal radiotherapy; TACE, transarterial chemoembolization; TAI, transarterial infusion chemotherapy. 
vs DEB-TACE group (4.34, 2.22-8.50), TACE + PEI vs DEB-TACE group (3.68, 1.31-10.31), TACE + RT vs DEB-TACE group (3.43, 1.22-9.65), and TACE + RFA vs RFA group (1.67, 1.06-2.56) (Figure 6).

\section{Secondary efficacy outcome - DFS}

\section{1-year PFS}

The network meta-analysis suggested that, compared with TACE treatment alone, TACE + PEI was ranked best for improving 1-year PFS (5.93, 1.39-25.34), followed by TACE + PAI (17.65, 0.00-2.23e+6), TACE + amiodarone (2.92, 0.53-16.16), TACE + RFA (3.05, 0.00-1.04e+5),
PAI (5.92, 0.00-7.22e+6), SIRT (1.67, 0.21-13.52), RFA (1.72, 0.00-5.86e+4), TACE + sorafenib (1.31, 0.752.27), TACE + IFN (1.25, 0.52-2.96), DEB-TACE (1.18, 0.30-4.65), and TACE + brivanib (1.09, 0.51-2.33).

When we assessed the comparative efficacy, TACE+PEI was superior to all other treatment arms. However, except for the TACE+PEI arm, the other treatment arms did not reach significance. Treatment arms were comparable to each other in terms of the effects of 1-year PFS, and no significant differences were found (Figure 7).

\section{2-year PFS}

The network meta-analysis suggested that, compared with TACE treatment alone, TACE + brivanib was ranked

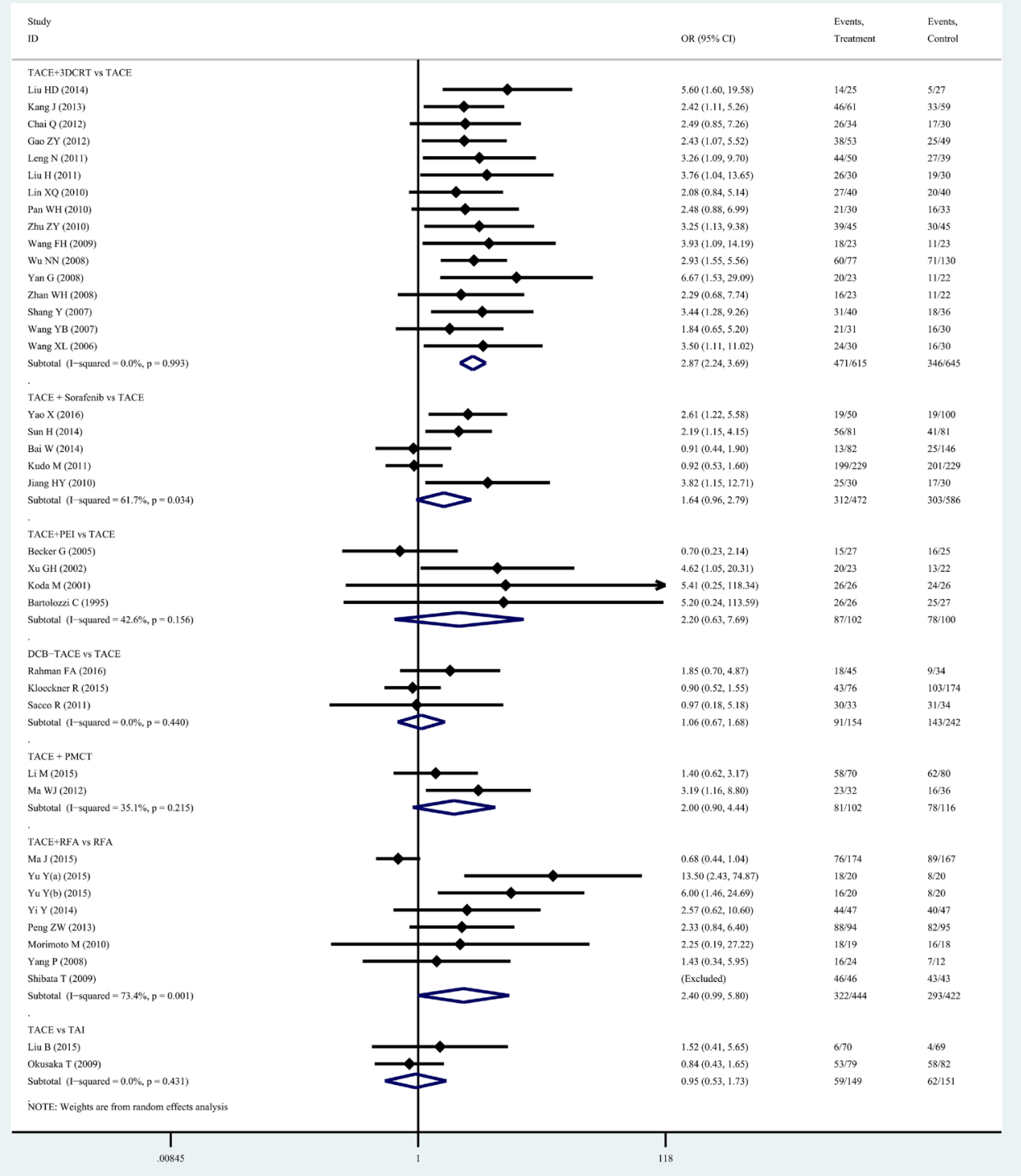

Figure 5: Overall 1-year survival for patients with unresectable hepatocellular carcinoma. Abbreviations: 3DCRT, threedimension eonformal radiotherapy; DEB-TACE, drug-eluting bead transarterial chemoembolization; PEI, percutaneous ethanol injection; PMCT, percutaneous microwave coagulation therapy; RFA, radiofrequency ablation; TACE, transarterial chemoembolization; TAI, transarterial infusion chemothera. 
best for improving 2-year PFS $(30.72,0.00-5.81 \mathrm{e}+10)$, followed by TACE + PAI $(26.79,0.00-1.49 \mathrm{e}+7), \mathrm{TACE}+$ RFA (8.15, 0.00 to $5.29 \mathrm{e}+6)$, TACE + amiodarone $(8.03$, $0.80-80.28)$, RFA (6.72, 0.00-4.37e+6), PAI (6.48, 0.00$3.59 \mathrm{e}+6)$, TACE + PEI $(3.79,1.22-11.81)$, DEB-TACE $(3.53,1.56-8.01)$, TACE + IFN $(2.59,1.49-4.49)$, and TACE + sorafenib $(0.95,0.58-1.56)$.
When we assessed the comparative efficacy, TACE + brivanib was superior to all other treatment arms. However, except for the TACE + PEI arm, TACE + IFN arm, and DEB-TACE arm, the other treatment arms did not reach significance. Treatment arms were comparable with each other for improving 2-year PFS, with a significant difference found in the TACE + sorafenib vs TACE +

\begin{tabular}{|c|c|c|c|c|c|c|c|c|c|c|c|c|c|c|c|c|}
\hline $\begin{array}{c}\text { TACE } \\
+ \text { Brivanib } \\
\end{array}$ & \begin{tabular}{|c|}
32.77 \\
$(0.00,7.58 \mathrm{e}+6)$ \\
\end{tabular} & \begin{tabular}{|c|}
34.01 \\
$(0.00,8.03 \mathrm{e}+6)$ \\
\end{tabular} & - & \begin{tabular}{|c|}
42.47 \\
$(0.00,9.94 \mathrm{e}+6)$ \\
\end{tabular} & \begin{tabular}{c|}
0.08 \\
$(0.00,19167.08)$ \\
\end{tabular} & \begin{tabular}{|c|}
0.08 \\
$(0.00,22257.98)$ \\
\end{tabular} & \begin{tabular}{|c|}
18.60 \\
$(0.00,4.44 \mathrm{e}+6)$ \\
\end{tabular} & $\begin{array}{c}0.06 \\
(0.00,15168.18) \\
\end{array}$ & \begin{tabular}{|c|}
0.13 \\
$(0.00,2.67 \mathrm{e}+5)$ \\
\end{tabular} & \begin{tabular}{|c|}
7.03 \\
$(0.00,5.50 \mathrm{e}+6)$ \\
\end{tabular} & \begin{tabular}{c|}
0.09 \\
$(0.00,20512.55)$ \\
\end{tabular} & \begin{tabular}{c|}
0.10 \\
$(0.00,23666.74)$ \\
\end{tabular} & - & \begin{tabular}{|c|}
4.25 \\
$(0.00,3.32 \mathrm{e}+6)$ \\
\end{tabular} & & $\begin{array}{c}1.44 \\
(0.00,2.90 \mathrm{e}+6)\end{array}$ \\
\hline $\begin{array}{c}0.85 \\
(0.47,1.55) \\
\end{array}$ & TACE & $\begin{array}{c}0.96 \\
(0.46,2.02) \\
\end{array}$ & - & $\begin{array}{c}0.77 \\
(0.43,1.39) \\
\end{array}$ & $\begin{array}{c}2.64 \\
(1.13,6.21)^{*} \\
\end{array}$ & \begin{tabular}{c|}
2.51 \\
$(0.21,29.81)$ \\
\end{tabular} & $\begin{array}{c}1.76 \\
(0.70,4.41) \\
\end{array}$ & $\begin{array}{c}2.13 \\
(1.24,3.66)^{*}\end{array}$ & $\begin{array}{c}4.34 \\
(0.00,14418.54) \\
\end{array}$ & \begin{tabular}{c|c}
4.66 \\
$(0.01,4058.47)$ \\
\end{tabular} & \begin{tabular}{|c|}
2.84 \\
$(1.21,6.63)^{*}$ \\
\end{tabular} & $\begin{array}{c}3.35 \\
(2.41,4.66)^{*} \\
\end{array}$ & - & \begin{tabular}{|c|}
7.72 \\
$(0.01,6731.08)$ \\
\end{tabular} & - & $\begin{array}{c}47.16 \\
(0.01,1.57 \mathrm{e}+5)\end{array}$ \\
\hline $\begin{array}{c}2.65 \\
(1.35,5.17)^{*}\end{array}$ & $\begin{array}{c}1.10 \\
(0.59,2.06)\end{array}$ & TAI & - & $\begin{array}{c}1.25 \\
(0.49,3.21) \\
\end{array}$ & $\begin{array}{c}2.74 \\
(0.89,8.49)\end{array}$ & $\begin{array}{c}2.61 \\
(0.20,34.47) \\
\end{array}$ & $\begin{array}{c}0.55 \\
(0.17,1.78)\end{array}$ & $\begin{array}{c}2.21 \\
(0.88,5.52) \\
\end{array}$ & $\begin{array}{c}4.50 \\
(0.00,15433.88)\end{array}$ & \begin{tabular}{c|c|}
4.84 \\
$(0.01,4364.47)$
\end{tabular} & $\begin{array}{c}2.95 \\
(0.96,9.08)\end{array}$ & $\begin{array}{c}3.48 \\
(1.55,7.82)^{*}\end{array}$ & - & \begin{tabular}{c|c|}
8.01 \\
$(0.01,7238.55)$
\end{tabular} & 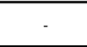 & $\begin{array}{c}48.95 \\
(0.01,1.68 \mathrm{e}+5)\end{array}$ \\
\hline $\begin{array}{c}3.04 \\
(0.55,16.94) \\
\end{array}$ & $\begin{array}{c}0.96 \\
(0.18,5.25) \\
\end{array}$ & \begin{tabular}{|c|}
11.56 \\
$(0,02,7612.10)$ \\
\end{tabular} & SIRT & - & - & - & - & - & - & - & - & - & - & - & - & - \\
\hline $\begin{array}{c}0.67 \\
(0.31,1.42) \\
\end{array}$ & $\begin{array}{c}1.28 \\
(0.80,2.03) \\
\end{array}$ & $\begin{array}{c}0.86 \\
(0.40,1.88) \\
\end{array}$ & $\begin{array}{c}0.75 \\
(0.15,3.87) \\
\end{array}$ & DCB-TACE & 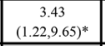 & $\begin{array}{c}3.26 \\
(0.26,41.36) \\
\end{array}$ & $\begin{array}{c}2.28 \\
(0.77,6.78) \\
\end{array}$ & $\begin{array}{c}2.76 \\
(1.24,6.12)^{*}\end{array}$ & \begin{tabular}{c|}
5.62 \\
$(0.00,19045.33)$ \\
\end{tabular} & \begin{tabular}{|c|}
6.04 \\
$(0.01,5376.63)$ \\
\end{tabular} & \begin{tabular}{|c|}
3.68 \\
$(1.31,10.31)^{* *}$ \\
\end{tabular} & $\begin{array}{c}4.34 \\
(2.22,8.50)^{*} \\
\end{array}$ & - & \begin{tabular}{|c|}
10.00 \\
$(0.01,8917.25)$ \\
\end{tabular} & - & $\begin{array}{c}61.12 \\
(0.02,2.07 \mathrm{e}+5) \\
\end{array}$ \\
\hline $\begin{array}{c}25.47 \\
02,26472.42) \\
\end{array}$ & $\begin{array}{c}1.62 \\
(0.77,3.43) \\
\end{array}$ & \begin{tabular}{c|c|}
19.73 \\
$(0.02,20554.90$ \\
\end{tabular} & $\begin{array}{c}22.66 \\
(0.02,27910.71) \\
\end{array}$ & $\begin{array}{c}1.27 \\
(0.53,3.07) \\
\end{array}$ & $\begin{array}{c}\text { TACE } \\
+\mathrm{RT} \\
\end{array}$ & \begin{tabular}{|c|}
1.05 \\
$(0.08,14.38)$ \\
\end{tabular} & $\begin{array}{c}1.50 \\
(0.43,5.25) \\
\end{array}$ & $\begin{array}{c}1.24 \\
(0.45,3.42) \\
\end{array}$ & \begin{tabular}{|c|}
1.64 \\
$(0.00,5681.05)$ \\
\end{tabular} & \begin{tabular}{|c|}
0.57 \\
$(0.00,518.01)$ \\
\end{tabular} & $\begin{array}{c}0.93 \\
(0.28,3.10) \\
\end{array}$ & $\begin{array}{c}0.79 \\
(0.32,1.97) \\
\end{array}$ & - & \begin{tabular}{|c|}
2.94 \\
$(0.00,313.09)$ \\
\end{tabular} & - & $\begin{array}{c}16.61 \\
(0.00,57373.91) \\
\end{array}$ \\
\hline $\begin{array}{c}2.60 \\
(1.15,5.88)^{*} \\
\end{array}$ & $\begin{array}{c}1.65 \\
(0.34,8.08) \\
\end{array}$ & $\begin{array}{c}2.01 \\
(0.87,4.65) \\
\end{array}$ & $\begin{array}{c}2.31 \\
(0.39,13.85) \\
\end{array}$ & $\begin{array}{c}1.29 \\
(0.25,6.77) \\
\end{array}$ & \begin{tabular}{c|}
13.20 \\
$(0.01,15890.01)$ \\
\end{tabular} & \begin{tabular}{|c|} 
TACE \\
+ Amiodarone \\
\end{tabular} & $\begin{array}{c}1.43 \\
(0.10,19.93) \\
\end{array}$ & $\begin{array}{c}0.85 \\
(0.07,10.63) \\
\end{array}$ & $\begin{array}{c}1.73 \\
(0.00,8009.47)\end{array}$ & \begin{tabular}{|c|}
0.54 \\
$(0.00,689.35)$ \\
\end{tabular} & \begin{tabular}{|c|}
1.13 \\
$(0.08,15.40)$ \\
\end{tabular} & $\begin{array}{c}0.75 \\
(0.06,9.09)\end{array}$ & . & \begin{tabular}{|c|}
3.03 \\
$(0.00,416.63)$ \\
\end{tabular} & - & $\begin{array}{c}18.76 \\
(0.00,87234.92)\end{array}$ \\
\hline $\begin{array}{c}1.68 \\
(0.70,4.06) \\
\end{array}$ & $\begin{array}{c}1.73 \\
(0.74,4.04) \\
\end{array}$ & $\begin{array}{c}0.55 \\
(0.08,3.70) \\
\end{array}$ & $\begin{array}{c}7.35 \\
(0.01,4955.91) \\
\end{array}$ & $\begin{array}{c}1.36 \\
(0.52,3.57) \\
\end{array}$ & $\begin{array}{c}12.55 \\
(0.01,1.34 \mathrm{e}+4) \\
\end{array}$ & $\begin{array}{c}1.28 \\
(0.46,3.53) \\
\end{array}$ & $\begin{array}{l}\text { TACE } \\
+ \text { SBRT } \\
\end{array}$ & $\begin{array}{c}1.21 \\
(0.42,3.50) \\
\end{array}$ & \begin{tabular}{c|c|}
2.46 \\
$(0.00,8577.45)$ \\
\end{tabular} & \begin{tabular}{c|c|}
2.64 \\
$(0.00,2431.54)$ \\
\end{tabular} & 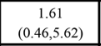 & $\begin{array}{c}1.90 \\
(0.72,5.04) \\
\end{array}$ & - & \begin{tabular}{|c|}
4.38 \\
$(0.00,4032.74)$ \\
\end{tabular} & - & $\begin{array}{c}26.76 \\
(0.01,93427.01)\end{array}$ \\
\hline $\begin{array}{c}3.47 \\
(1.38,8.77)^{*} \\
\end{array}$ & $\begin{array}{c}2.22 \\
(1.27,3.88)^{*}\end{array}$ & $\begin{array}{c}2.69 \\
(1.05,6.91)^{*} \\
\end{array}$ & $\begin{array}{c}3.09 \\
(0.49,19.49) \\
\end{array}$ & $\begin{array}{c}1.74 \\
(0.84,360) \\
\end{array}$ & $\begin{array}{c}0.73 \\
(0.29,1.86) \\
\end{array}$ & \begin{tabular}{c|}
1.80 \\
$(0.32,10.26)$ \\
\end{tabular} & $\begin{array}{c}1.71 \\
(0.57,5.16) \\
\end{array}$ & $\begin{array}{l}\text { TACE } \\
\text { +IFN } \\
\end{array}$ & \begin{tabular}{|c|}
2.04 \\
$(0.00,6894.53)$ \\
\end{tabular} & \begin{tabular}{|c|}
0.46 \\
$(0.00,405.22)$ \\
\end{tabular} & $\begin{array}{c}1.33 \\
(0.49,3.65) \\
\end{array}$ & $\begin{array}{c}0.63 \\
(0.34,1.20) \\
\end{array}$ & - & \begin{tabular}{|c|}
3.57 \\
$(0.00,244.92)$ \\
\end{tabular} & - & $\begin{array}{c}22.18 \\
(0.01,75096.99) \\
\end{array}$ \\
\hline $\begin{array}{c}0.56 \\
(0.11,2.82) \\
\end{array}$ & $\begin{array}{c}9.16 \\
(0.01,9.27 \mathrm{e}+3) \\
\end{array}$ & \begin{tabular}{c|c|}
13.27 \\
$(0.02,10430.42)$ \\
\end{tabular} & $\begin{array}{c}0.74 \\
(0.40,1.40) \\
\end{array}$ & $\begin{array}{c}7.18 \\
(0.01,7.36 \mathrm{e}+3) \\
\end{array}$ & $\begin{array}{c}5.64 \\
(0.01,5932.80) \\
\end{array}$ & $\begin{array}{c}1.34 \\
(0.54,3.29) \\
\end{array}$ & $\begin{array}{c}3.33 \\
(1.30,8.33)^{*} \\
\end{array}$ & $\begin{array}{c}0.55 \\
(0.20,1.53) \\
\end{array}$ & PAI & $\begin{array}{c}0.93 \\
(0.00,17575.89)\end{array}$ & \begin{tabular}{|c|}
1.53 \\
$(0,00,5267.43)$ \\
\end{tabular} & \begin{tabular}{|c|}
1.30 \\
$(0.00,4318.03)$ \\
\end{tabular} & - & $\begin{array}{c}1.79 \\
(0.00,10619.85) \\
\end{array}$ & - & $\begin{array}{c}11.11 \\
(4.34,25.00) \\
\end{array}$ \\
\hline $\begin{array}{c}0.31 \\
(0.00,197.71)\end{array}$ & $\begin{array}{c}9.46 \\
(0.01,6.05 \mathrm{e}+3)\end{array}$ & $\begin{array}{c}0.77 \\
(0.33,1.84)\end{array}$ & $\begin{array}{c}0.89 \\
(0.15,5.39)\end{array}$ & $\begin{array}{c}7.42 \\
.01,4.81 \mathrm{e}+3) \\
\end{array}$ & $\begin{array}{c}2.30 \\
(0.00,21976.82)\end{array}$ & \begin{tabular}{c|c|}
0.23 \\
$(0.00,153.42)$
\end{tabular} & $\begin{array}{c}0.49 \\
(0.18,1.39)\end{array}$ & $\begin{array}{c}0.31 \\
(0.00,207.41)\end{array}$ & \begin{tabular}{|c|}
0.96 \\
$(0.01,6537.9)$ \\
\end{tabular} & RFA & \begin{tabular}{|c|}
0.61 \\
$(0.00,553.94)$ \\
\end{tabular} & \begin{tabular}{c|c|}
0.72 \\
$(0.00,628.94)$
\end{tabular} & - & \begin{tabular}{c|c|}
1.67 \\
$(1.06,2.56)^{*}$
\end{tabular} & - & $\begin{array}{c}10.12 \\
(0.00,1.91 \mathrm{e}+5)\end{array}$ \\
\hline $\begin{array}{c}1.90 \\
(0.73,4.94) \\
\end{array}$ & \begin{tabular}{c|c|}
2.97 \\
$(1.46,6.02)^{*}$ \\
\end{tabular} & $\begin{array}{c}1.47 \\
(0.56,3.90) \\
\end{array}$ & $\begin{array}{c}1.69 \\
(0.26,10.84) \\
\end{array}$ & $\begin{array}{c}2.32 \\
(1.00,5.42) *\end{array}$ & $\begin{array}{c}7.33 \\
(0.01,7681.25) \\
\end{array}$ & $\begin{array}{c}0.99 \\
(0.17,5.71) \\
\end{array}$ & $\begin{array}{c}0.94 \\
(0.30,2.90) \\
\end{array}$ & $\begin{array}{c}0.73 \\
(0.29,1.86) \\
\end{array}$ & $\begin{array}{c}13.39 \\
(0.01,14112.53) \\
\end{array}$ & \begin{tabular}{c|c|}
0.17 \\
$(0.00,114.23)$ \\
\end{tabular} & $\begin{array}{l}\text { TACE } \\
+ \text { PEI }\end{array}$ & $\begin{array}{c}0.85 \\
(0.34,2.11) \\
\end{array}$ & - & \begin{tabular}{|c|}
2.70 \\
$(0.00,334.81)$ \\
\end{tabular} & - & $\begin{array}{c}1.53 \\
(0.00,5267.43) \\
\end{array}$ \\
\hline $\begin{array}{c}3.42 \\
(1.79,6.51)^{*}\end{array}$ & $\begin{array}{c}2.92 \\
(2.28,3.73)^{*}\end{array}$ & $\begin{array}{c}1.50 \\
(0.27,8.25) \\
\end{array}$ & $\begin{array}{c}1.72 \\
(0.17,17.60)\end{array}$ & $\begin{array}{c}2.29 \\
(1.35,3.87)^{*}\end{array}$ & $\begin{array}{c}7.45 \\
(0.01,7577.90) \\
\end{array}$ & $\begin{array}{c}0.76 \\
(0.41,1.40) \\
\end{array}$ & $\begin{array}{c}0.95 \\
(0.16,5.76) \\
\end{array}$ & $\begin{array}{c}1.02 \\
(0.48,2.15) \\
\end{array}$ & $\begin{array}{c}1.35 \\
(0.25,7.27)\end{array}$ & \begin{tabular}{c|c|}
0.17 \\
$(0.00,133.40)$ \\
\end{tabular} & $\begin{array}{c}0.56 \\
(0.25,1.22) \\
\end{array}$ & $\begin{array}{c}\text { TACE } \\
+3 \text { DCRT }\end{array}$ & - & \begin{tabular}{|c|}
2.33 \\
$(0.00,380.14)$ \\
\end{tabular} & - & \begin{tabular}{c|c}
14.08 \\
$(0.00,47033.24)$
\end{tabular} \\
\hline $\begin{array}{c}3.57 \\
(1.61,8.33)^{*}\end{array}$ & $\begin{array}{c}3.10 \\
(1.77,5.45)^{*}\end{array}$ & $\begin{array}{c}2.78 \\
(1.22,6.67)^{*}\end{array}$ & $\begin{array}{c}3.22 \\
(0.54,20.00)\end{array}$ & $\begin{array}{c}2.43 \\
(1.17,5.05)^{*}\end{array}$ & $\begin{array}{c}7.01 \\
(0.01,7250.91) \\
\end{array}$ & $\begin{array}{c}0.53 \\
(0.10,2.87)\end{array}$ & $\begin{array}{c}0.56 \\
(0.20,1.54) \\
\end{array}$ & $\begin{array}{c}0.72 \\
(0.32,1.58)\end{array}$ & $\begin{array}{c}2.11 \\
(0.71,6.30) \\
\end{array}$ & $\begin{array}{c}3.05 \\
(0.00,1991.31) \\
\end{array}$ & $\begin{array}{c}0.96 \\
(0.39,2.36) \\
\end{array}$ & $\begin{array}{c}0.94 \\
(0.51,1.74)\end{array}$ & $\begin{array}{c}\text { TACE } \\
+ \text { Sorafenib }\end{array}$ & & 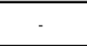 & \\
\hline $\begin{array}{c}0.23 \\
(0.00,147.12) \\
\end{array}$ & \begin{tabular}{|c|}
12.73 \\
$(0.02 .8 .16 \mathrm{e}+3)$ \\
\end{tabular} & \begin{tabular}{c|c|}
8.59 \\
$(0.01,5646.04)$ \\
\end{tabular} & $\begin{array}{c}9.86 \\
(0.01,7736.74) \\
\end{array}$ & $\begin{array}{c}9.98 \\
(0.02,6.48 \mathrm{e}+3) \\
\end{array}$ & $\begin{array}{c}1.71 \\
(0.00,16347.43)\end{array}$ & $\begin{array}{c}0.17 \\
(0.00,114.16)\end{array}$ & $\begin{array}{c}5.46 \\
(0.01,3675.91) \\
\end{array}$ & $\begin{array}{c}0.23 \\
(0.00,154.34)\end{array}$ & $\begin{array}{c}0.09 \\
(0.00,59.19) \\
\end{array}$ & \begin{tabular}{|c|}
0.07 \\
$(0.00,44.04)$ \\
\end{tabular} & \begin{tabular}{|c|}
0.13 \\
$(0.00,85.00)$ \\
\end{tabular} & $\begin{array}{c}0.13 \\
(0.00,99.26)\end{array}$ & $\begin{array}{c}4.10 \\
(0.01,2684.72) \\
\end{array}$ & $\begin{array}{l}\text { TACE } \\
+ \text { RFA } \\
\end{array}$ & - & \begin{tabular}{c|c|}
6.11 \\
$(0.00,1.16 \mathrm{e}+5)$
\end{tabular} \\
\hline $\begin{array}{c}1.25 \\
(0.61,2.63) \\
\end{array}$ & $\begin{array}{c}3.65 \\
(1.83,7.31)^{*} \\
\end{array}$ & $\begin{array}{c}3.84 \\
(0.61,25.00)^{*} \\
\end{array}$ & $\begin{array}{c}3.48 \\
(0.01,2307.23) \\
\end{array}$ & $\begin{array}{c}2.86 \\
(1.24,6.60) \\
\end{array}$ & \begin{tabular}{c|c}
5.95 \\
$(0.01,6227.54)$ \\
\end{tabular} & $\begin{array}{c}0.61 \\
(0.25,1.48) \\
\end{array}$ & $\begin{array}{c}0.64 \\
(0.22,1.82) \\
\end{array}$ & $\begin{array}{c}0.81 \\
(0.30,2.19) \\
\end{array}$ & $\begin{array}{c}0.87 \\
(0.14,5.33) \\
\end{array}$ & \begin{tabular}{|c|}
4.35 \\
$(1.72,11.11)^{*}$ \\
\end{tabular} & $\begin{array}{c}0.44 \\
(0.16,1.23) \\
\end{array}$ & $\begin{array}{c}0.45 \\
(0.08,2.55) \\
\end{array}$ & $\begin{array}{c}1.18 \\
(0.48,2.88) \\
\end{array}$ & \begin{tabular}{|c|}
2.59 \\
$(0.00,1711.32)$ \\
\end{tabular} & $\begin{array}{c}\text { TACE } \\
+ \text { PMCT } \\
\end{array}$ & \\
\hline $\begin{array}{c}10.73 \\
01,11128.7\end{array}$ & $\begin{array}{c}21.74 \\
(0.02,2.21 \mathrm{e}+3)\end{array}$ & $\begin{array}{c}8.31 \\
(0.01,8641.09)\end{array}$ & $\begin{array}{c}9.55 \\
(0.01,11733.96)\end{array}$ & $\begin{array}{c}17.04 \\
(0.02,1.75 \mathrm{e}+4)\end{array}$ & $\begin{array}{c}4.82 \\
(0.00,1.54 \mathrm{e}+4)\end{array}$ & $\begin{array}{c}5.56 \\
(0.00,6680.29)\end{array}$ & $\begin{array}{c}5.29 \\
(0.00,5618.81)\end{array}$ & $\begin{array}{c}4.13 \\
(0.00,4267.51)\end{array}$ & $\begin{array}{c}0.42 \\
(0.17,1.05)\end{array}$ & $\begin{array}{c}0.97 \\
(0.00,9242.86)\end{array}$ & \begin{tabular}{c|}
3.09 \\
$(0.00,3229.13)$
\end{tabular} & $\begin{array}{c}3.14 \\
(0.00,3185.66)\end{array}$ & $\begin{array}{c}2.95 \\
(0.00,3048.21)\end{array}$ & $\begin{array}{c}0.72 \\
(0.00,6875.29\end{array}$ & $\begin{array}{c}2.51 \\
(0.00,2618.01)\end{array}$ & $\begin{array}{l}\text { TACE } \\
\text { +PAI }\end{array}$ \\
\hline
\end{tabular}

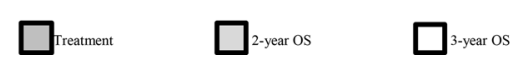

Figure 6: Network meta-analysis of 2-year OS and 3-year OS. Treatments are reported in order of survival rate ranking according to SUCRA of treatment response. Comparisons should be read from left to right. The estimate is located at the intersection of the column-defining treatment and the row-defining treatment. For OS, an OR value above 1 favors the treatment arm. ${ }^{*}$ Result with significant difference. Abbreviations: 3DCRT, three-dimension eonformal radiotherapy; DEB-TACE, drug-eluting bead transarterial chemoembolization; IFN, interferon-a; PAI, percutaneous acetic acid injection; PEI,percutaneous ethanol injection; PMCT, percutaneous microwave coagulation therapy; RFA, radiofrequency ablation; RT, radiotherapy therapy; SBRT, stereotactic body radiation therapy; SIRT, selective internal radiotherapy; TACE, transarterial chemoembolization; TAI, transarterial infusion chemotherapy.

\begin{tabular}{|c|c|c|c|c|c|c|c|c|c|c|c|}
\hline TACE & $\begin{array}{c}30.72 \\
(0.00,5.81 \mathrm{e}+10) \\
\end{array}$ & $\begin{array}{c}3.53 \\
(1.56,8.01) * \\
\end{array}$ & $\begin{array}{c}2.59 \\
(1.49,4.49) * \\
\end{array}$ & $\begin{array}{c}0.95 \\
(0.58,1.56) \\
\end{array}$ & $\begin{array}{c}6.72 \\
(0.00,4.37 \mathrm{e}+6) \\
\end{array}$ & - & $\begin{array}{c}6.48 \\
(0.00,3.59 \mathrm{e}+6) \\
\end{array}$ & \begin{tabular}{c|}
8.15 \\
$(0.00,5.29 \mathrm{e}+6)$ \\
\end{tabular} & $\begin{array}{c}8.03 \\
(0.80,80.28) \\
\end{array}$ & $\begin{array}{c}26.79 \\
(0.00,1.49 \mathrm{e}+7) \\
\end{array}$ & $\begin{array}{c}3.79 \\
(1.22,11.81) *\end{array}$ \\
\hline $\begin{array}{c}1.09 \\
(0.51,2.33) \\
\end{array}$ & $\begin{array}{c}\text { TACE } \\
+ \text { Brivanib } \\
\end{array}$ & $\begin{array}{c}8.70 \\
(0.00,1.66 \mathrm{e}+10) \\
\end{array}$ & $\begin{array}{c}0.08 \\
(0.00,1.60 \mathrm{e}+8) \\
\end{array}$ & $\begin{array}{c}32.32 \\
(0.00,6.13 \mathrm{e}+10) \\
\end{array}$ & $\begin{array}{c}4.57 \\
(0.00,2.12 \mathrm{e}+11) \\
\end{array}$ & - & $\begin{array}{c}0.21 \\
(0.00,7.36 \mathrm{e}+9) \\
\end{array}$ & $\begin{array}{c}3.77 \\
(0.00,1.75 \mathrm{e}+11) \\
\end{array}$ & $\begin{array}{c}0.26 \\
(0.00,5.45 \mathrm{e}+8) \\
\end{array}$ & $\begin{array}{c}0.87 \\
(0.00,3.05 \mathrm{e}+10) \\
\end{array}$ & $\begin{array}{c}0.12 \\
(0.00,2.40 \mathrm{e}+8) \\
\end{array}$ \\
\hline $\begin{array}{c}1.18 \\
(0.30,4.65) \\
\end{array}$ & $\begin{array}{c}0.92 \\
(0.19,4.43) \\
\end{array}$ & DCB-TACE & $\begin{array}{c}0.73 \\
(0.27,1.97) \\
\end{array}$ & $\begin{array}{c}0.27 \\
(0.10,0.70) * \\
\end{array}$ & $\begin{array}{c}1.90 \\
(0.00,1.26 \mathrm{e}+6) \\
\end{array}$ & - & $\begin{array}{c}1.84 \\
(0.00,1.04 \mathrm{e}+6) \\
\end{array}$ & $\begin{array}{c}2.31 \\
(0.00,1.53 \mathrm{e}+6) \\
\end{array}$ & $\begin{array}{c}2.27 \\
(0.20,26.17) \\
\end{array}$ & $\begin{array}{c}7.59 \\
(0.00,4.30 \mathrm{e}+6) \\
\end{array}$ & $\begin{array}{c}1.07 \\
(0.27,4.36) \\
\end{array}$ \\
\hline $\begin{array}{c}1.25 \\
(0.52,2.96) \\
\end{array}$ & $\begin{array}{c}1.14 \\
(0.36,3.62) \\
\end{array}$ & $\begin{array}{c}1.06 \\
(0.21,5.34) \\
\end{array}$ & $\begin{array}{l}\text { TACE } \\
+ \text { IFN } \\
\end{array}$ & $\begin{array}{c}2.72 \\
(1.30,5.72) * \\
\end{array}$ & $\begin{array}{c}0.39 \\
(0.00,2.53 \mathrm{e}+5) \\
\end{array}$ & - & $\begin{array}{c}2.50 \\
(0.00,1.40 \mathrm{e}+6) \\
\end{array}$ & $\begin{array}{c}0.32 \\
(0.00,2.08 \mathrm{e}+5) \\
\end{array}$ & $\begin{array}{c}0.32 \\
(0.03,3.44) \\
\end{array}$ & $\begin{array}{c}10.34 \\
(0.00,5.82 \mathrm{e}+6) \\
\end{array}$ & $\begin{array}{c}1.47 \\
(0.41,5.17) \\
\end{array}$ \\
\hline $\begin{array}{c}1.31 \\
(0.75,2.27) \\
\end{array}$ & $\begin{array}{c}0.84 \\
(0.33,2.13) \\
\end{array}$ & $\begin{array}{c}1.11 \\
(0.25,4.84) \\
\end{array}$ & $\begin{array}{c}0.96 \\
(0.34,2.66) \\
\end{array}$ & $\begin{array}{c}\text { TACE } \\
+ \text { Sorafenib } \\
\end{array}$ & \begin{tabular}{|c|}
7.07 \\
$(0.00,4.63 \mathrm{e}+6)$ \\
\end{tabular} & - & $\begin{array}{c}6.82 \\
(0.00,3.81 \mathrm{e}+6) \\
\end{array}$ & $\begin{array}{c}8.57 \\
(0.00,5.61 \mathrm{e}+6) \\
\end{array}$ & $\begin{array}{c}8.45 \\
(0.80,89.00) \\
\end{array}$ & $\begin{array}{c}28.19 \\
(0.00,1.58 \mathrm{e}+7) \\
\end{array}$ & $\begin{array}{c}3.99 \\
(1.16,13.78) * \\
\end{array}$ \\
\hline $\begin{array}{c}1.72 \\
(0.00,5.86 \mathrm{e}+4) \\
\end{array}$ & $\begin{array}{c}0.64 \\
(0.00,2.21 \mathrm{e}+4) \\
\end{array}$ & $\begin{array}{c}1.45 \\
(0.00,5.35 \mathrm{e}+4) \\
\end{array}$ & \begin{tabular}{c|}
0.73 \\
$(0.00,2.55 \mathrm{e}+4)$ \\
\end{tabular} & $\begin{array}{c}1.32 \\
(0.00,4.54 \mathrm{e}+4) \\
\end{array}$ & RFA & - & $\begin{array}{c}0.96 \\
(0.00,2.80 \mathrm{e}+07) \\
\end{array}$ & $\begin{array}{c}0.82 \\
(0.46,1.47) \\
\end{array}$ & $\begin{array}{c}1.20 \\
(0.00,9.00 \mathrm{e}+5) \\
\end{array}$ & $\begin{array}{c}3.99 \\
(0.00,1.16 \mathrm{e}+8) \\
\end{array}$ & $\begin{array}{c}0.56 \\
(0.00,3.82 \mathrm{e}+5) \\
\end{array}$ \\
\hline $\begin{array}{c}1.67 \\
(0.21,13.52) \\
\end{array}$ & $\begin{array}{c}0.65 \\
(0.07,6.02) \\
\end{array}$ & $\begin{array}{c}1.42 \\
(0.12,17.23) \\
\end{array}$ & $\begin{array}{c}0.75 \\
(0.08,7.15) \\
\end{array}$ & $\begin{array}{c}1.28 \\
(0.15,11.12) \\
\end{array}$ & $\begin{array}{c}1.03 \\
(0.00,4.15 \mathrm{e}+4) \\
\end{array}$ & SIRT & - & - & - & - & - \\
\hline $\begin{array}{c}5.92 \\
(0.00,7.22 \mathrm{e}+5) \\
\end{array}$ & $\begin{array}{c}5.43 \\
(0.00,6.76 \mathrm{e}+5) \\
\end{array}$ & $\begin{array}{c}5.01 \\
(0.00,6.54 \mathrm{e}+4) \\
\end{array}$ & $\begin{array}{c}4.75 \\
(0.00,5.94 \mathrm{e}+5) \\
\end{array}$ & $\begin{array}{c}4.54 \\
(0.00,5.58 \mathrm{e}+5) \\
\end{array}$ & $\begin{array}{c}3.45 \\
(0.00,5.72 \mathrm{e}+6) \\
\end{array}$ & \begin{tabular}{c|c|}
3.54 \\
$(0.00,5.02 \mathrm{e}+5)$ \\
\end{tabular} & PAI & \begin{tabular}{c|c|}
0.80 \\
$(0.00,2.31 \mathrm{e}+7)$ \\
\end{tabular} & $\begin{array}{c}0.81 \\
(0.00,5.19 \mathrm{e}+5) \\
\end{array}$ & $\begin{array}{c}4.16 \\
(1.49,11.11) *\end{array}$ & $\begin{array}{c}1.71 \\
(0.00,9.85 e+5) \\
\end{array}$ \\
\hline $\begin{array}{c}3.05 \\
(0.00,1.04 \mathrm{e}+5)\end{array}$ & $\begin{array}{c}0.36 \\
(0.00,1.24 \mathrm{e}+4)\end{array}$ & $\begin{array}{c}2.59 \\
(0.00,9.51 \mathrm{e}+4)\end{array}$ & $\begin{array}{c}0.41 \\
(0.00,1.43 \mathrm{e}+4)\end{array}$ & $\begin{array}{c}2.34 \\
(0.00,8.07 \mathrm{e}+4)\end{array}$ & $\begin{array}{c}0.56 \\
(0.28,1.12)\end{array}$ & $\begin{array}{c}1.82 \\
(0.00,7.39 \mathrm{e}+4)\end{array}$ & $\begin{array}{c}1.94 \\
(0.00,3.22 \mathrm{e}+6)\end{array}$ & $\begin{array}{l}\text { TACE } \\
+ \text { RFA }\end{array}$ & $\begin{array}{c}0.99 \\
(0.00,7.41 \mathrm{e}+5)\end{array}$ & $\begin{array}{c}3.29 \\
(0.00,9.57 \mathrm{e}+7)\end{array}$ & $\begin{array}{c}0.47 \\
(0.00,3.14 \mathrm{e}+5)\end{array}$ \\
\hline $\begin{array}{c}2.92 \\
(0.53,16.16)\end{array}$ & $\begin{array}{c}2.68 \\
(0.41,17.41)\end{array}$ & $\begin{array}{c}2.47 \\
(0.28,22.13)\end{array}$ & $\begin{array}{c}0.43 \\
(0.06,2.90)\end{array}$ & $\begin{array}{c}2.24 \\
(0.37,13.49)\end{array}$ & $\begin{array}{c}1.70 \\
(0.00,6.52 \mathrm{e}+4)\end{array}$ & $\begin{array}{c}1.75 \\
(0.12,25.94)\end{array}$ & $\begin{array}{c}2.03 \\
(0.00,2.74 \mathrm{e}+5) \\
\end{array}$ & $\begin{array}{c}0.96 \\
(0.00,3.67 \mathrm{e}+4)\end{array}$ & $\begin{array}{c}\text { TACE } \\
+ \text { Amiodarone }\end{array}$ & $\begin{array}{c}3.34 \\
(0.00,2.16 \mathrm{e}+6)\end{array}$ & $\begin{array}{c}0.47 \\
(0.04,6.15)\end{array}$ \\
\hline $\begin{array}{c}17.65 \\
(0.00,2.23 \mathrm{e}+6)\end{array}$ & $\begin{array}{c}16.19 \\
(0.00,2.08 \mathrm{e}+6)\end{array}$ & $\begin{array}{c}14.95 \\
(0.00,2.02 \mathrm{e}+6)\end{array}$ & $\begin{array}{c}14.16 \\
(0.00,1.83 \mathrm{e}+6)\end{array}$ & $\begin{array}{c}13.52 \\
(0.00,1.72 \mathrm{e}+6)\end{array}$ & $\begin{array}{c}10.28 \\
(0.00,1.75 \mathrm{e}+7)\end{array}$ & $\begin{array}{c}10.55 \\
(0.00,1.55 \mathrm{e}+6)\end{array}$ & $\begin{array}{c}0.34 \\
(0.03,3.61)\end{array}$ & $\begin{array}{c}5.78 \\
(0.00,9.87 \mathrm{e}+6)\end{array}$ & $\begin{array}{c}6.04 \\
(0.00,8.45 \mathrm{e}+5)\end{array}$ & $\begin{array}{l}\text { TACE } \\
+ \text { PAI }\end{array}$ & $\begin{array}{c}7.06 \\
(0.00,4.09 \mathrm{e}+6)\end{array}$ \\
\hline $\begin{array}{c}5.93 \\
(1.39,25.34) * \\
\end{array}$ & $\begin{array}{c}5.44 \\
(1.06,28.01)\end{array}$ & $\begin{array}{c}5.02 \\
(0.68,36.98) \\
\end{array}$ & $\begin{array}{c}4.76 \\
(0.88,25.78) \\
\end{array}$ & $\begin{array}{c}4.54 \\
(0.96,21.47) \\
\end{array}$ & $\begin{array}{c}3.45 \\
(0.00,1.28 \mathrm{e}+5) \\
\end{array}$ & $\begin{array}{c}3.54 \\
(0.28,45.09) \\
\end{array}$ & $\begin{array}{c}1.00 \\
(0.00,1.32 \mathrm{e}+5) \\
\end{array}$ & $\begin{array}{c}1.94 \\
(0.00,7.22 \mathrm{e}+4) \\
\end{array}$ & $\begin{array}{c}2.03 \\
(0.22,19.11) \\
\end{array}$ & $\begin{array}{c}2.98 \\
(0.00,4.05 \mathrm{e}+5) \\
\end{array}$ & $\begin{array}{l}\text { TACE } \\
+ \text { PEI } \\
\end{array}$ \\
\hline
\end{tabular}

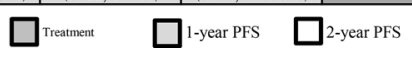

Figure 7: Network meta-analysis of 1-year PFS and 2-year PFS. Treatments are reported in order of survival rate ranking according to SUCRA of treatment response. Comparisons should be read from left to right. The estimate is located at the intersection of the column-defining treatment and the row-defining treatment. For PFS, an OR value above 1 favors the treatment arm. ${ }^{*}$ Result with significant difference. Abbreviation: DEB-TACE, drug-eluting bead transarterial chemoembolization; IFN, interferon-a; PAI, percutaneous acetic acid injection; PEI, percutaneous ethanol injection; RFA, radiofrequency ablation; SIRT, selective internal radiotherapy; TACE, transarterial chemoembolization. 
IFN group (2.72, 1.30-5.72), TACE + PEI vs TACE + sorafenib group $(3.99,1.16-13.78)$, and TACE+PAI vs PAI group (4.16,1.49 to 11.11) (Figure 7).

\section{Safety outcomes}

Table 3 summarizes the results of the toxicity associated with the different treatment groups. We found that combined therapy with sorafenib may elevate the AST level $(5.541,2.04-14.48)$ and ALT level $(4.48,1.66-$ 12.11), and increase the occurrence of clinical symptoms, such as pain/abdominal distension, hand-foot-skin reaction, hypertension, and fatigue. Moreover, the TACE + RFA vs RFA group may increase the occurrence of nausea and vomiting $(1.65,1.01-2.69)$. The other treatment groups were safe, with mostly moderate- to high-quality evidence according to GRADE assessment.

\section{DISCUSSION}

The network meta-analysis represents the most comprehensive synthesis of data for currently available data for the treatment for combination therapy with TACE with TACE monotherapy. We combined direct and indirect evidence from 48 clinical trials (50 analyses) comparing 19 different interventions on over five thousand patients with unresectable HCC to identify the best combination treatment with TACE. First, we found that most combination therapy arms were significantly more effective than TACE monotherapy in terms of treatment response. TACE + PEI had superior treatment efficacy, followed by TACE + sorafenib and TACE + 3DCRT (Figure 3). Second, TACE + 3DCRT was significantly more effective than TACE monotherapy in 6-month OS and 1-year OS (Figure 4), and similar results could be found in the standardized meta-analysis of 1-year OS (Figure 5). Third, we found that TACE + PAI and TACE + PMCT are the most significant effective combination therapies with TACE arms for 2-year OS, in comparison with other combination therapies and monotherapies; similar results could also be found for 3-year OS (Figure 6). Finally, TACE + PEI and TACE + PAI have displayed a leading efficacy tendency for 1-year PFS and 2-year DFS, in comparison with other treatment arms (Figure 7). Moreover, when considering the safety results, sorafenib and RFA may increase the occurrence of adverse effects (Table 3). In summary, in comparison with other types of combination therapy arms, TACE + 3DCRT, TACE + PEI, TACE + PMCT, TACE + PAI, and TACE + sorafenib were the more effective methods; however, because the adverse effects of sorafenib were serious, the use of TACE + sorafenib arm is not recommended.

This study extends the findings from primary clinical trials and previous meta-analyses by systematically synthesizing the efficacy data [28-75]. The meta-analysis differs from those in earlier studies in several ways.
First, the main objective of the study was to identify the best combination treatment with TACE, including 19 arms, whereas the previous pairwise studies included only two combination treatments of TACE + RFA/PEI vs monotherapy $[18,25-26]$ with a standardized metaanalysis. Second, a network meta-analysis was used to directly and indirectly compare the treatment response, OS rate, and DFS rate, to determine the most appropriate types of combination therapy with TACE, while the previous article only considered the OS rate results [27]. Finally, the adverse effects of groups $(n \geq 2)$ were considered to observe the safety indicators.

This review followed the guidelines for conducting rigorous systematic reviews and network meta-analyses [56-58]. To identify as many relevant reports as possible, and to decrease the risk of bias, a comprehensive search strategy was designed. Based on these considerations, we observed no evidence of publication bias by statistical assessment. Combination therapy significantly increased the treatment response, 6-month OS, 1-year OS, 2-year OS, 3-year OS, 1-year DFS, and 2-year DFS (Figures $3-7)$. The results could be verified in the published review [14]. As for the safety section, sorafenib-associated adverse effects were more frequent in the combination therapy group, although it may improve the treatment response, OS, and DFS for unresectable HCC patients. The conclusions of the published articles [22, 79] are similar to those of our research. Moreover, DEB-TACE may have a higher OS rate and a higher DFS rate in patients with HCC than TACE. This conclusion can also be confirmed in published articles [80], which probably mean that DEB-TACE combined with other percutaneous local-ablation and targeted therapy drugs may further improve the survival rate.

This network meta-analysis had several limitations that merit further discussion. First, the review was restricted to trials involving patients with unresectable HCC. We excluded studies in which the patient was diagnosed with HCC, including a significant number of patients seen in the real world. Similarly, we excluded studies that only provided descriptive data. We did this to reduce heterogeneity and inconsistency among the trials included in this network meta-analysis; however, we acknowledge that this restricts the external validity of the results. Furthermore, in some cases, the original language of the publications could not be obtained, which reduced the number of trials we included and may have impacted the accuracy of the results. Furthermore, positive results are likely to be published, while negative results are not likely to be shared [81]. An additional limitation of standardize outcomes is their extensive heterogeneity (Figure 5), which indicated substantial variability in the outcomes of the included studies, although this was often because of the presence of heterogeneity in the baseline outcomes (Table 1) and differences observed in the trial design, Child-Pugh, and tumor stage of each study. 


\begin{tabular}{|c|c|c|c|c|c|c|c|}
\hline \multirow[t]{2}{*}{ Adverse Events } & \multirow[t]{2}{*}{ Treatment arms } & \multicolumn{3}{|c|}{ All } & \multicolumn{3}{|c|}{ Grade $\geq 3$} \\
\hline & & Analysis (patients) & OR $(95 \%$ CI $)$ & $P, I^{2}$ & Analysis (patients) & OR $(95 \%$ CI $)$ & $P, I^{2}$ \\
\hline \multicolumn{8}{|l|}{ Hematological system } \\
\hline Leucopenia & TACE + 3DCRT vs TACE & $3(127 / 115)$ & $0.76(0.18,3.16)$ & $0.011,77.6 \%$ & $1(40 / 36)$ & $0.44(0.04,5.02)$ & - \\
\hline \multirow[t]{2}{*}{ Thrombocytopenia } & TACE + 3DCRT vs TACE & $1(40 / 36)$ & $0.56(0.14,2.15)$ & - & $1(40 / 36)$ & $0.58(0.09,3.68)$ & - \\
\hline & $\mathrm{TACE}+$ Sorafenib & $2(311 / 391)$ & $4.12(0.38,44.69)$ & $0.008,85.7 \%$ & $1(229 / 227)$ & $11.40(1.46,89.08)^{*}$ & \\
\hline \multicolumn{8}{|l|}{ Clinical biochemistry } \\
\hline Total bilirubin & TACE +3DCRT vs TACE & $1(40 / 36)$ & $3.43(0.99,11.85)$ & - & & & \\
\hline AST & $\mathrm{TACE}+$ Sorafenib & $1(229 / 227)$ & $5.541(2.04,14.48)^{*}$ & - & $1(229 / 227)$ & $4.13(1.15,14.83)^{\circ}$ & - \\
\hline \multirow[t]{2}{*}{ ALT } & TACE + 3DCRT vs TACE & $2(101 / 95)$ & $1.80(0.64,5.03)$ & $0.166,48.0 \%$ & $1(40 / 36)$ & $1.84(0.16,21.22)$ & - \\
\hline & $\mathrm{TACE}+$ Sorafenib & $1(229 / 227)$ & $4.48(1.66,12.11)^{\circ}$ & & $1(229 / 227)$ & $4.07(0.86,19.39)$ & - \\
\hline Albumin & TACE + 3DCRT vs TACE & $1(40 / 36)$ & $3.43(0.99,11.85)$ & - & - & & \\
\hline \multicolumn{8}{|l|}{ Clinical symptoms } \\
\hline \multirow[t]{3}{*}{ Nausea/ vomiting } & TACE + 3DCRT vs TACE & $3(127 / 115)$ & $0.29(0.05,1.74)$ & $0.001,84.2 \%$ & $1(40 / 36)$ & $0.88(0.29,2.64)$ & - \\
\hline & $\mathrm{TACE}+$ Sorafenib & $1(81 / 81)$ & $0.64(0.28,1.48)$ & - & - & & \\
\hline & TACE + RFA vs RFA & $2(141 / 142)$ & $1.65(1.01 .2 .69)^{*}$ & $0.902,0.0 \%$ & $2(141 / 142)$ & $3.03(0.59,15.55)$ & $0.669,0.0 \%$ \\
\hline \multirow[t]{2}{*}{ Fever } & TACE + 3DCRT vs TACE & $2(114 / 108)$ & $1.13(0.62,2.05)$ & $0.910,0.0 \%$ & & & \\
\hline & TACE + RFA vs RFA & $2(141 / 142)$ & $1.41(0.85,2.33)$ & $0.911,0.0 \%$ & $2(141 / 142)$ & $2.41(0.43,13.69)$ & $0.431,0.0 \%$ \\
\hline \multirow[t]{3}{*}{ Pain/abdominal distension } & TACE + 3DCRT vs TACE & $2(114 / 108)$ & $1.38(0.75,2.55)$ & $0.644,0.0 \%$ & & & \\
\hline & $\mathrm{TACE}+$ Sorafenib & $2(311 / 391)$ & $29.00(0.86,983.24)$ & $0.017,82.5 \%$ & $2(311 / 391)$ & $8.98(1.61,50.18)^{\circ}$ & $0.540,0.0 \%$ \\
\hline & TACE+RFA vs RFA & $2(141 / 142)$ & $1.19(0.53,2.70)$ & - & $2(141 / 142)$ & $1.00(0.06,16.47)$ & - \\
\hline \multirow{2}{*}{$\begin{array}{l}\text { Upper gastrointestinal } \\
\text { hemorrhage }\end{array}$} & $\mathrm{TACE}+$ Sorafenib & $1(82 / 164)$ & $0.64(0.24,1.68)$ & - & & & \\
\hline & TACE + RFA vs RFA & $2(141 / 142)$ & $3.06(0.31,29.86)$ & $1.000,0.0 \%$ & - & - & - \\
\hline Hand-foot-skin reaction. & $\mathrm{TACE}+$ Sorafenib & $3(392 / 472)$ & $15.58(0.77,313.76)$ & $0.000,95.7 \%$ & $2(311 / 391)$ & $31.40(3.30,299.23)^{*}$ & $\begin{array}{l}0.275 \\
16.20 \%\end{array}$ \\
\hline Hypertension & $\mathrm{TACE}+$ Sorafenib & $3(392 / 472)$ & $3.65(1.10,12.17)^{*}$ & $0.020,74.3 \%$ & $1(229 / 227)$ & $15.84(2.08,120.96)^{*}$ & - \\
\hline Fatigue & TACE + Sorafenib & $1(82.164)$ & $107.92(6.43,1811.3)^{*}$ & & & & \\
\hline
\end{tabular}

Additional clinical trials of combination therapy with TACE should include larger samples and be robust and randomized to confirm the effects and toxicity of combination therapy on patient-relevant or disease-specific outcomes, particularly in cancer patients with unresectable HCC. Future studies should ensure that appropriate methods are used for randomization, blinding, and intent to-treat. Furthermore, trials should assess outcomes using standardized or prescribed measures at similar time points. The analyses of individual data will be valuable for further exploration. More normative studies should be utilized in future network meta-analyses.

The findings of this comprehensive network metaanalysis provide some evidence that combination therapy with TACE may improve treatment response, 6-month OS, 1-year OS, 2-year OS, 3-year OS, 1-year DFS, and 2-year OS, which did not increase the occurrence of adverse effects, except sorafenib. On a local scale, patients with unresectable HCC should be encouraged to accept combination therapy with TACE, especially TACE + 3DCRT, TACE + PEI, TACE + PMCT, and TACE + PAI. In the clinical treatment of unresectable HCC patients, combination therapy with TACE can be used as the firstline treatment.

\section{MATERIALS AND METHODS}

\section{Search strategy and selection criteria}

This systematic review was performed with an a priori established protocol (PROSPERO CRD42017071102) [82], and the meta-analysis was performed following the Preferred Reporting Items for the Systematic Reviews and Meta-analyses (PRISMA) statement, the PRISMA network statement, and the Cochrane Collaboration recommendations [76-78].

We considered large-scale clinical trials of patients with unresectable HCC and searched PubMed, EMBase, the Cochrane Library, and Chinese National Knowledge Infrastructure for eligible trials form the very beginning of the databases to June 2017, comparing any of the following treatments: combination treatment with TACE, or TACE alone (see details in Supplementary Table 4). Trials of TACE or combined treatment groups within trials of TACE that did not have sufficient data for analysis were not considered. We also excluded trials published only as abstracts (with no additional data available from other sources). No language restrictions were applied. We then screened the reference lists of all obtained articles to avoid missing relevant trials. 
We included the following treatment arms: threedimensional conformal radiotherapy, drug-eluting bead transarterial chemoembolization, high-dose hepatic arterial infusion chemotherapy, interferon-a, percutaneous acetic acid injection, percutaneous ethanol injection, percutaneous microwave coagulation therapy, radiofrequency ablation, radiotherapy therapy, stereotactic body radiation therapy, selective internal radiotherapy, transarterial chemoembolization, and transarterial infusion chemotherapy. Trials involving patients with a primary diagnosis of HCC, and no surgery can be made.

\section{Data extraction and quality assessment}

Two investigators (XH and YHP) selected trials independently, and the third investigator ( $\mathrm{ZG}$ or WHM) independently reviewed the main reports and supplementary materials and extracted the relevant information from the included trials with the predefined data extraction sheet. Data on efficacy and safety were abstracted from original studies. We extracted trial design, trial size, tumor stage, tumor size, Child-Pugh, ECOG status, $\mathrm{HBV}, \mathrm{HCV}$, the number of tumors, the details of treatment arms, the type of outcome (efficacy and safety), and outcome data for each time-point of interest. Whenever necessary, we approximated the means and measures of dispersion from figures in the original studies [83]. We extracted the results from intention-to-treat analyses whenever possible.

The risk of bias of the individual studies was assessed using the Newcastle-Ottawa scale score [84]. The scale is based on the Newcastle-Ottawa scale's "yes" or "no" answers to the following criteria: (1) Is the case definition adequate? (2) Is there representativeness of the cases? (3) Is there selection of controls? (4) Is there a definition of controls? (5) Is there comparability of cases and controls? (6) Is there ascertainment of exposure? (7) Is the same method of ascertainment used for cases and controls? (8) Is there a non-response rate? A system analysis of studies was performed that excluded those with scores less than 5. Any discrepancies were resolved by consensus and arbitration by a panel of investigators within the review team.

\section{Outcomes}

The primary efficacy outcome was treatment response. Local tumor response was measured according to the modified criteria for response evaluation in solid tumors (mRECIST) [85]; mRECIST defined the treatment response into four main categories: complete response (CR), partial response (PR), progressive disease (PD), and stable disease (SD). CR corresponds to disappearance of any intra-tumoral arterial enhancement in all target lesions, and PR corresponds to at least a $30 \%$ decrease in the sum of diameters of viable (enhancement in the arterial phase) target lesions, taking as a reference the baseline sum of the diameters of target lesions. PD is defined as an increase of at least $20 \%$ in the sum of the diameters of viable target lesions, taking as a reference the smallest sum of the diameters of viable target lesions recorded at the start of treatment; SD is defined as any cases that do not qualify for either PR or PD.

We also considered the 6-month, 1-year, 2-year, and 3-year overall survival (OS) as the primary efficacy outcomes of unresectable HCC associated with different treatment arms with or without TACE use. Our secondary efficacy outcome was 1-year to 2-year disease-free survival (DFS) associated with different treatment arms.

Our primary safety outcomes were hematological system and clinical biochemistry toxicity; the former including leucopenia and thrombocytopenia and the latter including total bilirubin, AST, and ALT, which could be stratified by all adverse events or grade $\geq 3$. Our secondary safety outcomes were clinical symptoms toxicity, including nausea/vomiting, fever, pain/abdominal distension, upper gastrointestinal hemorrhage, hand-footskin reaction, hypertension, fatigue, and stomatitis, which also could be stratified by all adverse events or grade $\geq 3$.

\section{Data synthesis and statistical analysis}

We defined studies reporting multiple treatments and controls as sub-studies (marked as a/b) to avoid doublecounting and mistreating data. First, a direct meta-analysis was performed with random-effects models because they are likely the most appropriate and conservative methodology to account for between-trial heterogeneity for each comparison [86-87]. To estimate pooled odds ratios (ORs) and 95\% confidence intervals (95\% CIs) heterogeneity was incorporated within and between studies, with STATA v14.0. Statistical heterogeneity was assessed with $P$ values and $I 2$ statistics, with values higher than 50\% indicating substantial heterogeneity [88]. We also plotted a comparison-adjusted funnel plot for the network meta-analysis to detect the presence of any dominant publication bias in the network meta-analysis.

Second, we conducted a random-effects network meta-analysis using STATA v14.0. We summarized the results of the network meta-analysis with OR and their credible intervals (CrI) [89]. A common heterogeneity parameter was assumed for all comparisons; we also assessed global heterogeneity using $P$ values and the $I^{2}$ statistic.

The relative efficacy and safety of each treatment resulted from the combination of the direct evidence between the two treatment arms and the indirect evidence derived from the network meta-analysis, which are assumed to be coherent [86]. Inconsistency between direct and indirect sources of evidence was statistically 
assessed globally (by comparison of the fit and parsimony of consistency and inconsistency models) and locally (by calculation of the difference between direct and indirect estimates in all closed loops in the network) [90]. When a direct connection between two treatment arms was not available, the result was obtained only from indirect evidence.

We estimated the ranking probabilities for all treatments of being at each possible rank for each treatment arm. The treatment hierarchy was summarized and reported as the surface under the cumulative ranking curve (SUCRA) [91], ranging from 1, indicating that the treatment has a high likelihood of being best, to 0 , indicating that the treatment has a high likelihood of being worst. A high SUCRA score corresponds to a higher ranking of survival rate from cancer compared with other treatments.

\section{Quality of evidence}

We assessed the quality of evidence for our primary outcomes according to the Grading of Recommendations Assessment, Development and Evaluation (GRADE) system using GRADEpro GDT [92-93]. The GRADE system assesses risk of bias (study limitations), imprecision, inconsistency, indirectness of study results, and publication bias (classifying each as high, moderate, low, or very low) across the body of evidence to derive an overall summary of the quality of evidence.

\section{Patient involvement}

No patients were involved in setting the research question or the outcome measures, nor were they involved in developing plans for the design or implementation of the study. No patients were asked for advice on the interpretation or presentation of results. There are no plans to disseminate the results of the research to study participants or the relevant patient community.

\section{Author contributions}

$\mathrm{XH}$ and YHP conceived and designed the experiment; $\mathrm{XH}, \mathrm{TST}$ and $\mathrm{YXL}$ performed the experiments; XH, YHP, WHM and ZG analyzed the data; XH, YHP, WXM and ZY contributed materials/analysis tools; $\mathrm{XH}$ and YHP wrote the paper. All authors approved the final version of the article, including the authorship list.

\section{ACKNOWLEDGMENTS}

We thank the authors of the primary studies for providing their data and other critical information. Additionally, the authors would like to thank the researchers and participants for their valuable contributions to this article.

\section{CONFLICTS OF INTEREST}

The authors have declared that there is no competing interest.

\section{FUNDING}

Supported by: National Natural Science Foundation of China, No. 81471761 and No.81501568; Tianjin Program to Support Science and Technology Research, No.15ZCZDSY00890.

\section{REFERENCES}

1. Forner A, Llovet JM, Bruix J. Hepatocellular carcinoma. Lancet. 2012; 379:1245-55.

2. Siegel RL, Miller KD, Jemal A. Cancer Statistics, 2017. CA Cancer J Clin. 2017; 67:7-30.

3. Jia H, Yang Q, Wang T, Cao Y, Jiang QY, Ma HD, Sun HW, Hou MX, Yang YP, Feng F. Rhamnetin induces sensitization of hepatocellularcarcinoma cells to a small molecularkinase inhibitor or chemotherapeutic agents. Biochim Biophys Acta. 2016; 1860:1417-30.

4. Otaegui-Arrazola A, Amiano P, Elbusto A, Urdaneta E, Martinez-Lage P. Diet, cognition, and Alzheimer's disease: food for thought. Eur J Nutr. 2014; 53:1-23.

5. Bruix J, Sherman M, American Association for the Study of Liver D. Management of hepatocellular carcinoma: an update. Hepatology. 2011; 53:1020-1022.

6. Jiang BG, Wang N, Huang J, Yang Y, Sun LL, Pan ZY, Zhou WP. Tumor SOCS3 methylation status predicts the treatment response to TACE and prognosis in HCC patients. Oncotarget. 2017; 8:28621-28627. https://doi. org/10.18632/oncotarget.16157.

7. Ma X, Li RS, Wang J, Huang YQ, Li PY, Wang J, Su HB, Wang RL, Zhang YM, Liu HH, Zhang CE, Ma ZJ, Wang JB, et al. The Therapeutic Efficacy and Safety of Compound Kushen Injection Combined with Transarterial Chemoembolization in Unresectable Hepatocellular Carcinoma: An Update Systematic Review and MetaAnalysis. Front Pharmacol. 2016; 7:70.

8. Llovet JM, Bruix J. Systematic review of randomized trials for unresectable hepatocellular carcinoma: chemoembolization improves survival. Hepatology. 2003; $37: 429-42$.

9. Sun JH, Zhou TY, Zhang YL, Zhou GH, Nie CH, Zhu TY, Chen SQ, Wang BQ, Ye S, Shen Y, Guo H, Wang WL, Zheng SS. Efficacy of transcatheter arterial chemoembolization for liver metastases arising from pancreatic cancer. Oncotarget. 2017; 8:39746-39755. https://doi.org/10.18632/oncotarget.14642.

10. Wang FS, Fan JG, Zhang Z, Gao B, Wang HY. The global burden of liver disease: the major impact of China. Hepatology. 2014; 60:2099-108. 
11. Lewandowski RJ, Kulik LM, Riaz A, Senthilnathan S, Mulcahy MF, Ryu RK, Ibrahim SM, Sato KT, Baker T, Miller FH, Omary R, Abecassis M, Salem R. A comparative analysis of transarterial downstaging for hepatocellular carcinoma: chemoembolization versus radioembolization. Am J Transplant. 2009; 9:1920-1928.

12. Jansen MC, van Hillegersberg R, Chamuleau RA, van Delden OM, Gouma DJ, van Gulik TM. Outcome of regional and local ablative therapies for hepatocellular carcinoma: a collective review. Eur J Surg Oncol. 2005; $31: 331-47$.

13. Chua TC, Liauw W, Saxena A, Chu F, Glenn D, Chai A, Morris DL. Systematic review of neoadjuvant transarterial chemoembolization for resectable hepatocellular carcinoma. Liver Int. 2009; 30:166-74.

14. Qi X, Zhao Y, Li H, Guo X, Han G. Management of hepatocellular carcinoma: an overview of major findings from meta-analyses. Oncotarget. 2016; 7:34703-51. https:// doi.org/10.18632/oncotarget.9157.

15. Yu TZ, Zhang W, Liu QX, Li WH, Ma JQ, Zhang ZH, Yang MJ, Wang JH, Chen B, Zeng SC, Luo JJ, Liu LX, Yan ZP. Endovascular brachytherapy combined with portal vein stenting and transarterial chemoembolization improves overall survival of hepatocellular carcinoma patients with main portal vein tumor thrombus. Oncotarget. 2017; 8:12108-12119. https://doi.org/10.18632/oncotarget.14520.

16. Wang C, Wang H, Yang W, Hu K, Xie H, Hu KQ, Bai W, Dong Z, Lu Y, Zeng Z, Lou M, Wang H, Gao X, et al. Multicenter randomized controlled trial of percutaneous cryoablation versus radiofrequency ablation in hepatocellular carcinoma. Hepatology. 2015; 61:1579-90.

17. Hocquelet A, Seror O, Blanc JF, Frulio N, Salut C, Nault JC, Trillaud H. Transarterial chemoembolization for early stage hepatocellular carcinoma decrease local tumor control and overall survival compared to radiofrequency ablation. Oncotarget. 2017; 8:32190-32200. https://doi. org/10.18632/oncotarget.12921.

18. Fu Y, Zhao X, Yun Q, Zhu X, Zhu Y, Li Q, Hu K, Wang J, Qiao Z. Transarterial chemoembolization (TACE) plus percutaneous ethanol injection (PEI) for the treatment of unresectable hepatocellular carcinoma: a meta-analysis of randomized controlled trials. Int J Clin Exp Med. 2015; 8:10388-400.

19. Granata V, Fusco R, Catalano O, Piccirillo M, De Bellis M, Izzo F, Petrillo A. Percutaneous ablation therapy of hepatocellular carcinoma with irreversible electroporation: MRI findings. AJR Am J Roentgenol. 2015; 204:1000-7.

20. Zhuang X, Wang Q, Wang N, Hou X, Zhang J, Chi H. Effects of combining transarterial chemoembolization with percutaneous microwave coagulation therapy for hepatocellular carcinoma abutting the diaphragm. Minim Invasive Ther Allied Technol. 2016; 25:107-12.

21. Kloth C, Thaiss WM, Kärgel R, Grimmer R, Fritz J, Ioanoviciu $\mathrm{SD}$, Ketelsen D, Nikolaou K, Horger M. Evaluation of Texture
Analysis Parameter for Response Prediction in Patients with Hepatocellular Carcinoma Undergoing Drug-eluting Bead Transarterial Chemoembolization (DEB-TACE) Using Biphasic Contrast-enhanced CT Image Data: Correlation with Liver Perfusion CT. Acad Radiol. 2017.

22. Zhang X, Wang K, Wang M, Yang G, Ye X, Wu M, Cheng $\mathrm{S}$. Transarterial chemoembolization (TACE) combined with sorafenib versus TACE for hepatocellular carcinoma with portal vein tumor thrombus: a systematic review and metaanalysis. Oncotarget. 2017; 8:29416-29427. https://doi. org/10.18632/oncotarget.15075.

23. Bodzin AS, Busutl RW. Hepatocellular carcinoma: Advances in diagnosis, management, and long term outcome. World J Hepatol. 2015; 7:1157-1167.

24. Cheng AL, Kang YK, Chen Z, Tsao CJ, Qin S, Kim JS, Luo R, Feng J, Ye S, Yang TS, Xu J, Sun Y, Liang H, et al. Efficacy and safety of sorafenib in patients in the AsiaPacific region with advanced hepatocellular carcinoma: a phase III randomised, double-blind, placebo-controlled trial. Lancet Oncol. 2009; 10:25-34.

25. Wang W, Shi J, Xie WF. Transarterial chemoembolization in combination with percutaneous ablation therapy in unresectable hepatocellular carcinoma: a meta-analysis. Liver Int. 2010; 30:741-9.

26. Li L, Tian J, Liu P, Wang X, Zhu Z. Transarterial chemoembolization combination therapy vs monotherapy in unresectablehepatocellular carcinoma: a meta-analysis. Tumori. 2016; 2016:301-10.

27. Zhao J, Zhang H, Wei L, Xie S, Suo Z. Comparing the longterm efficacy of standard and combined minimally invasive procedures for unresectable HCC: a mixed treatment comparison. Oncotarget. 2017; 8:15101-15113. https://doi. org/10.18632/oncotarget.13145.

28. Rahman FA, Naidu J, Ngiu CS, Yaakob Y, Mohamed Z, Othman H, Jarmin R, Elias MH, Hamid NA, Mokhtar NM, Ali RR. Conventional versus Doxorubicin-Eluting Beads Transarterial Chemoembolization for Unresectable Hepatocellular Carcinoma: a Tertiary Medical Centre Experience in Malaysia. Asian Pac J Cancer Prev. 2016; 17:4037-41.

29. Yao X, Yan D, Zeng H, Liu D, Li H. Concurrent sorafenib therapy extends the interval to subsequent TACE for patients with unresectable hepatocellular carcinoma. J Surg Oncol. 2016; 113:672-7.

30. Zhang XC, He X, Wang YF, Zhao CN. Clinical effects of transcatheter arterial chemoembolization combined with sterotactic body radiation therapy on patients with unresectable hepatocellular carcinoma. Modern Oncology. 2016; 24:3437-3440.

31. Kloeckner R, Weinmann A, Prinz F, Pinto dos Santos D, Ruckes C, Dueber C, Pitton MB.Conventional transarterial chemoembolization versus drug-eluting bead transarterial chemoembolization for the treatment of hepatocellular carcinoma. BMC Cancer. 2015; 15:465. 
32. Li M, Yao L, Chen Y, Zhai PT, Liu QY, Yu GZ, Zhang QY, Yang GN. Efficiency of transcather hepatic arterial chemoembolization combined with microwave ablation in patients with advanced liver cancer. Modern Oncology. 2015; 23:2351-2355.

33. Liu B, Huang JW, Li Y, Hu BS, He X, Zhao W, Zheng YB, Lu LG. Arsenic trioxide transarterial chemoembolization with and without additional intravenous administration of arsenic trioxide in unresectable hepatocellular carcinoma with lung metastasis: a single-blind, randomized trial. J Cancer Res Clin Oncol. 2015; 141:1103-8.

34. Ma J, Wang JH. 131I-Labeled-Metuximab Plus Transarterial Chemoembolization in Combination Therapy for Unresectable Hepatocellular Carcinoma: Results from a Multicenter Phase IV Clinical Study. Asian Pac J Cancer Prev. 2015; 16:7441-7.

35. Pitton MB, Kloeckner R, Ruckes C, Wirth GM, Eichhorn W, Wörns MA, Weinmann A, Schreckenberger M, Galle PR, Otto G, Dueber C. Randomized comparison of selective internal radiotherapy (SIRT) versus drug-eluting bead transarterial chemoembolization (DEB-TACE) for the treatment of hepatocellular carcinoma. Cardiovasc Intervent Radiol. 2015; 38:352-60.

36. Yu Y, Hao J, Xu B, Wu YQ, Li MP, Hu SQ, Liao K, Pan M, Zuo JW, Lu SF. Effect of radiofrequency ablation combined with transcatheter artery chemoembolization and portal vein chemotherapy on primary hepatocellular carcinoma. Guangxi Medical Journal. 2015; 37:787-789.

37. Kudo M, Han G, Finn RS, Poon RT, Blanc JF, Yan L, Yang J, Lu L, Tak WY, Yu X, Lee JH, Lin SM, Wu C, et al. Brivanib as adjuvant therapy to transarterial chemoembolization in patients with hepatocellular carcinoma: A randomized phase III trial. Hepatology. 2014; 60:1697-707.

38. Liu HD, Ding G, Zhang M, Jiang F, Yuan CT, Liang HX, Xu J, Ni YF, Liao ZJ, Huang Q, Huang Y. Efficacy combined transcatheter arterial chemoembolization (TACE) and radiotherapy for advanced primary liver cancer. Modern Oncology. 2015; 23:1258-1261.

39. Sun H, Han WJ. Analvsis of curative effect of transcatheter arterial chemoembolization combined with sorafbnib fbr not operation resection of hepatoceuular carcinoma. Clin J Gastroenterol Hepatol. 2014; 23:486-488.

40. Yi Y, Zhang Y, Wei Q, Zhao L, Han J, Song Y, Ding Y, Lu G, Liu J, Ding H, Dai F, Tang X. Radiofrequency ablation or microwave ablation combined with transcatheter arterial chemoembolization in treatment of hepatocellular carcinoma by comparing with radiofrequency ablation alone. Chin J Cancer Res. 2014; 26:112-8.

41. Bai W, Wang YJ, Zhao Y, Qi XS, Yin ZX, He CY, Li RJ, Wu KC, Xia JL, Fan DM, Han GH. Sorafenib in combination with transarterial chemoembolization improves the survival of patients with unresectable hepatocellular carcinoma: a propensity score matching study. J Dig Dis. 2013; 14:181-90.

42. Kang J, Qi NX, Wang TQ, Cheng YX, Yang ZH, Li WY, Yang R. Clinical outcome of three dimensional conformal radiation combined with transarterial chemoembolization for unresectable primary hepatic cancer. Modern Oncology. 2013; 21:1834-1836.

43. Peng ZW, Zhang YJ, Chen MS, Xu L, Liang HH, Lin XJ, Guo RP, Zhang YQ, Lau WY. Radiofrequency ablation with or without transcatheter arterial chemoembolization in the treatment of hepatocellular carcinoma: a prospective randomized trial. J Clin Oncol. 2013; 31:426-32.

44. Chan Q. Transcatheter arterial chemoembolization therapy combined three-dimensional conformal radiotherapy Clinical observation of primary liver cancer. Journal of Chinese Physician. 2011; 13:181-187.

45. Gao Z, Zhang JB, Liu HT, Liu YL. Clinical study of hyperfractiommted three-dimensional conformal radiotherapy combined with transeatheter arterial chemoembolization for treating patients with inoperable primary liver cancer. Journal of Chinese physician education. 2012; 35:11-14.

46. Ma WJ, Guo SS, Zhang LC, Wei HM, Zhang L. Clinical review of transcatheter hepatic arterial chemoembolization combined with microwave ablation in patients with liver cancer. Modern Medicine Journal of China. 2012; 14:28-30.

47. Boulin M, Ciboulet A, Guiu B, Maillard E, Bonnetain F, Minello A, Gagnaire A, Lepage C, Krause D, Hillon P, Bedenne L, Cercueil JP, Chauffert B, et al. Randomised controlled trial of lipiodol transarterial chemoembolisation with or without amiodarone for unresectable hepatocellular carcinoma. Dig Liver Dis. 2011; 43:905-11.

48. Kudo M, Imanaka K, Chida N, Nakachi K, Tak WY, Takayama T, Yoon JH, Hori T, Kumada H, Hayashi N, Kaneko S, Tsubouchi H, Suh DJ, et al. Phase III study of sorafenib after transarterial chemoembolisation in Japanese and Korean patients with unresectable hepatocellular carcinoma. Eur J Cancer. 2011; 47:2117-27.

49. Leng N. The comparison of clinical efficacy of threedimensional conformal radiotherapy and simple interventional therapy for primary hepatic carcinoma. Chinese basic journal. 2011; 18:1966-1967.

50. Liu H, Wang SY. Therapeutic effect of three-dimensional conformal radiotherapy combined with hepatic arterial chemoembolization for primary liver cancer. China Prac Med. 2011; 6:56-57.

51. Pawlik TM, Reyes DK, Cosgrove D, Kamel IR, Bhagat N, Geschwind JF. Phase II trial of sorafenib combined with concurrent transarterial chemoembolization with drugeluting beads for hepatocellular carcinoma. J Clin Oncol. 2011; 29:3960-7.

52. Sacco R, Bargellini I, Bertini M, Bozzi E, Romano A, Petruzzi P, Tumino E, Ginanni B, Federici G, Cioni R, Metrangolo $\mathrm{S}$, Bertoni $\mathrm{M}$, Bresci $\mathrm{G}$, et al. Conventional versus doxorubicin-eluting bead transarterial chemoembolization for hepatocellular carcinoma. J Vasc Interv Radiol. 2011; 22:1545-52.

53. Jiang HY, Xie XD. Sorafenib combined with transcatheter arterial chemoembolization in the treatment of advanced hepatocellular carcinoma. Hainan Medical Journal. 2010; 21:6-9. 
54. Kim HY, Kim JD, Bae SH, Park JY, Han KH, Woo HY, Choi JY, Yoon SK, Jang BK, Hwang JS, Kim SG, Kim YS, Seo YS, et al. Korean Liver Cancer Study Group. A comparative study of high-dose hepatic arterial infusion chemotherapy and transarterial chemoembolization using doxorubicin for intractable, advanced hepatocellular carcinoma. Korean J Hepatol. 2010; 16:355-61.

55. Lin XQ, Huang ZL. Efficacy of three dimensional conformal radiotherapy combined with transcatheter arterial chemoembolization on primary hepatocellular carcinoma. Clin J Prim Med Pharm. 2010; 17:1751-1752.

56. Morimoto M, Numata K, Kondou M, Nozaki A, Morita S, Tanaka K. Midterm outcomes in patients with intermediatesized hepatocellular carcinoma: a randomized controlled trial for determining the efficacy of radiofrequency ablation combined with transcatheter arterial chemoembolization. Cancer. 2010; 116:5452-60.

57. Pan WH, Dong P, Chen HX, Sun HY. The effect of threedimensional conformal radiotherapy combined with hepatic arterial chemoembolization for primary liver cancer. China Foreign Medical Treatment. 2010; 30:82.

58. Zhu ZY, Xu YL, Sun AM, Lu W. A Study of Combined Therapy of Transcatheter Arterial Chemoembolization and Three Dimensional Conformal Radiotherapy for Unreseetable Hepatocellular Carcinoma. Chinese Journal of Medical Physics. 2010; 27:2084-2086.

59. Li M, Lu C, Cheng J, Zhang J, Cao C, Xu J, Xu J, Pan H, Zhong B, Tucker S, Wang D. Combination therapy with transarterial chemoembolization and interferon-alpha compared with transarterial chemoembolization alone for hepatitis B virus related unresectable hepatocellular carcinoma. J Gastroenterol Hepatol. 2009; 24:1437-44.

60. Okusaka T, Kasugai H, Shioyama Y, Tanaka K, Kudo M, Saisho H, Osaki Y, Sata M, Fujiyama S, Kumada T, Sato K, Yamamoto S, Hinotsu S, et al. Transarterial chemotherapy alone versus transarterial chemoembolization for hepatocellular carcinoma: a randomized phase III trial. J Hepatol. 2009; 51:1030-6.

61. Shibata T, Isoda H, Hirokawa Y, Arizono S, Shimada K, Togashi K. Small hepatocellular carcinoma: is radiofrequency ablation combined with transcatheter arterial chemoembolization more effective than radiofrequency ablation alone for treatment? Radiology. 2009; 252:905-13.

62. Wang FH. The combined therapy of TACE followed by 3DCRT for primary hepatocellular carcinoma. China Prac Med. 2009; 4:58-59.

63. Wu NN, Ma YL, Huang MJ, Ouyang X, Zhao C, Huang DJ. Transcatheter arterial chemoembolization combined with three-dimensional conformal radiotherapy in 77 patients with primary hepatic carcinoma. J Pract Med. 2008; 24:2573-2575.

64. Yan G, Zhan WH, Zhe H. Treatment of 45 cases of advanced hepatocellular carcinoma with the combined interventional therapy of three-dimensional conformal radiotherapy.
Chinese Radiation Oncology Branch Code Radiation Therapy Symposium (Northwest). 2008; 158-160.

65. Yang P, Liang M, Zhang Y, Shen B. Clinical application of a combination therapy of lentinan, multi-electrode RFA and TACE in HCC. Adv Ther. 2008; 25:787-94.

66. Zhan WH, Zhan YX, Yan G. Three - dimensional conformal radiotherapy combined with TACE in the treatment of advanced liver cancer: a report of 23 cases. Shandong Med. 2008; 48:51-52.

67. Shang Y, You GX, Xu HY, Chen MC. Treatment of 40 cases of primary liver cancer with transcatheter arterial chemoembolization combined with three - dimensional conformal radiotherapy.WCJD. 2007; 15:3140-3142.

68. Yang YZ, Wang TQ, Li S, Zhang YT. Three dimensional conformal radiation combined with fransarteriaI chemoembolizaton for primary hepatic cancer. Modern Oncology. 2007; 15:1646-1648.

69. Wang XL, Zheng LP, Xiong JP. A Clinical Study of Transcatheter Arterial Chemoembolization Combined with Three-dimensional Conformal Radiotherapy in Primary Hepatocellular Carcinoma. Practice Clinical Medicine. 2016; 7:21-23.

70. Becker G, Soezgen T, Olschewski M, Laubenberger J, Blum HE, Allgaier HP. Combined TACE and PEI for palliative treatment of unresectable hepatocellular carcinoma. World J Gastroenterol. 2005; 11:6104-9.

71. Liu MZ, Wang XS, Cai L, Gu MF, Liu H, Li Q, Cui NJ, Zhang YQ, Li GH, Li JQ. External Radiation and Combined Transcatheter Arterial Chemoembolization for Unresectable Primary Liver Cancer. Chinese Journal of Cancer. 2005; 24:82-86.

72. Huo TI, Huang YH, Wu JC, Chiang JH, Lee PC, Chang FY, Lee SD. Sequential transarterial chemoembolization and percutaneous acetic acid injection therapy versus repeated percutaneous acetic acid injection for unresectable hepatocellular carcinoma: a prospective study. Ann Oncol. 2003; 14:1648-53.

73. Xu GH, Wen HC, Li ZW, Wu HX, Cheng ZZ, Chen XH, Wu H, Huang JM, Wen YJ, Duan TH, Zhang GL, Xiao RJ. Evaluation of Hepatic Chemoembolization and Percutaneous Ethanol Injection in the Treatment of HCC. Journal of Clinical Radiology. 2002; 21:66-68.

74. Koda M, Murawaki Y, Mitsuda A, Oyama K, Okamoto K, Idobe Y, Suou T, Kawasaki H. Combination therapy with transcatheter arterial chemoembolization and percutaneousethanol injection compared with percutaneous ethanol injection alone for patients with smallhepatocellular carcinoma: a randomized control study. Cancer. 2001; 92:1516-24.

75. Bartolozzi C, Lencioni R, Caramella D, Vignali C, Cioni R, Mazzeo S, Carrai M, Maltinti G, Capria A, Conte PF. Treatment of large HCC: transcatheter arterial chemoembolization combined with percutaneous ethanol injection versus repeated transcatheter arterial chemoembolization. Radiology. 1995; 197:812-8. 
76. Moher D, Liberati A, Tetzlaff J, Altman DG; PRISMA Group. Preferred reporting items for systematic reviews and meta-analyses: the PRISMA statement. BMJ. 2009; 339:b2535.

77. Hutton B, Salanti G, Caldwell DM, Chaimani A, Schmid $\mathrm{CH}$, Cameron C, Ioannidis JP, Straus S, Thorlund K, Jansen JP, Mulrow C, Catalá-López F, Gøtzsche PC, et al. The PRISMA extension statement for reporting of systematic reviews incorporating network meta-analyses of health care interventions: checklist and explanations. Ann Intern Med. 2015; 162:777-784.

78. Cochrane handbook for systematic reviews of interventions. John Wiley \& Sons, 2011.

79. Yang M, Yuan JQ, Bai M, Han GH. Transarterial chemoembolization combined with sorafenib for unresectable hepatocellular carcinoma: a systematic review and meta-analysis. Mol Biol Rep. 2014; 41:6575-82.

80. Zou JH, Zhang L, Ren ZG, Ye SL. Efficacy and safety of cTACE versus DEB-TACE in patients with hepatocellular carcinoma: ameta-analysis. J Dig Dis. 2016; 17:510-517.

81. Zhang Y, Hou J, Feng F, Li D, Jiang Q, Li X, Zhao Q, Li BA. Genetic polymorphisms in human UDPglucuronosyltransferases $1 \mathrm{~A} 7$ and the risk of gastrointestinal carcinomas: A systematic review and network metaanalysis. Oncotarget. 2017; 8:66371-66381. https://doi. org/10.18632/oncotarget.18675.

82. PROSPERO, Centre for Reviews and Dissemination. Systematic Reviews: CRD's Guidance for Undertaking Reviews in Health Care (Internet). York, England: University of York. http://www.cdr.york.ac.uk/prospero), 2009.

83. Reichenbach S, Sterchi R, Scherer M, Trelle S, Bürgi E, Bürgi U, Dieppe PA, Jüni P. Meta-analysis: chondroitin for osteoarthritis of the knee or hip. Ann Intern Med. 2007; 146:580-590.

84. Wells GA, Shea B, O'Connell D, Peterson J, Welch V, Losos M, Tugwell P. The Newcastle-Ottawa Scale (NOS) for assessing the quality of nonrandomised studies in metaanalyses. Ottawa: Ottawa Hospital Research Institute. $2011 ; 2011$.

85. Lencioni R, Llovet JM. Modified RECIST (mRECIST) assessment for hepatocellular carcinoma. Semin Liver Dis. 2010; 30:52-60.

86. Dias S, Welton NJ, Sutton AJ, Caldwell DM, Lu G, Ades AE. Evidence synthesis for decision making 4: Inconsistency in networks of evidence based on randomized controlled trials. Med Decis Making. 2013; 33:641-56.

87. Mills EJ, Thorlund K, Ioannidis JP. Demystifying trial networks and network meta-analysis. BMJ. 2013; 346:f2914.

88. Higgins JP, Thompson SG, Deeks JJ, Altman DG. Measuring inconsistency in meta-analyses. BMJ. 2003; 327:557-60.

89. Salanti G, Higgins JP, Ades AE, Ioannidis JP. Evaluation of networks of randomized trials. Stat Methods Med Res. 2008; 17:279-301.

90. Chaimani A, Higgins JP, Mavridis D, Spyridonos P, Salanti G. Graphical tools for network meta-analysis in STATA. PLoS One. 2013; 8:e76654.

91. Salanti G, Ades AE, Ioannidis JP. Graphical methods and numerical summaries for presenting results from multipletreatment meta-analysis: an overview and tutorial. J Clin Epidemiol. 2011; 64:163-71.

92. Balshem H, Helfand M, Schünemann HJ, Oxman AD, Kunz R, Brozek J, Vist GE, Falck-Ytter Y, Meerpohl J, Norris S, Guyatt GH. GRADE guidelines: 3. Rating the quality of evidence. J Clin Epidemiol. 2011; 64:401-406.

93. Guyatt GH, Oxman AD, Vist GE, Kunz R, Falck-Ytter Y, Alonso-Coello P, Schünemann HJ; GRADE Working Group. GRADE: an emerging consensus on rating quality of evidence and strength of recommendations. BMJ. 2008; 336:924. 